\title{
LEARNING, INFORMATION PROCESSING AND ORDER SUBMISSION IN LIMIT ORDER MARKETS
}

\author{
CARL CHIARELLA, XUE-ZHONG HE AND LIJIAN WEI* \\ University of Technology Sydney, UTS Business School \\ PO Box 123, Broadway, NSW 2007, Australia
}

\begin{abstract}
By introducing a genetic algorithm learning with a classifier system into a limit order market, this paper provides a unified framework of microstructure and agent-based models of limit order markets that allows traders to determine their order submission endogenously according to market conditions. It examines how traders process and learn from market information and how the learning affects limit order markets. It is found that, measured by the average usage of different group of market information, trading strategies under the learning become stationary in long run. Also informed traders pay more attention to the last transaction sign while uninformed trader pay more attention to technical rules. Learning of uninformed traders improves market information efficiency, but not necessarily when informed traders learn. Opposite to the learning of informed traders, learning makes uninformed traders submit less aggressive limit orders and more market orders. Furthermore private values can have significant impact in short run, but not in long run. One implication is that the probability of informed trading (PIN) is positively related to the volatility and the bid-ask spread.
\end{abstract}

Key words: Limit order book, order submission, genetic algorithm learning, asymmetric information, probability of informed trading.

JEL Classification: G14, C63, D82

Date: March 26, 2015.

Acknowledgement: We thank the participants at the 2013 Workshop on Market Microstructure and Nonlinear Dynamics (University of Evry, France) and the 2013 Auckland Finance Conference (Auckland), the 2013 Quantitative Method in Finance conference (Sydney) and seminar participants in University of Technology, Sydney and Tianjin University. We thank Anthony Hall, Adrian Lee, Bruce Lehmann, Talis Putnins, Lei Shi, Jianxin Wang, Wei Zhang, Yongjie Zhang and Qiaoqiao Zhu for valuable suggestions. In particular, we would like to thank three anonymous referees for their very useful suggestions and comments which have improved the paper significantly. Financial support from the Australian Research Council (ARC) under the Discovery Grants (DP110104487, DP130103210) and the National Natural Science Foundation of China (NSFC) Grants $(71131007,71271145)$ is gratefully acknowledged. The usual caveat applies.

*Corresponding author.

Emails: Carl.Chiarella@uts.edu.au (Chiarella), Tony.He-1@uts.edu.au(He), weilijian@gmail.com (Wei). 
"It is the uninformed traders who provide the liquidity to the informed, and so understanding their behavior can provide substantial insight and intuition into the trading process...(an) open question is what traders can learn from other pieces of market data, such as prices"

-O'Hara (2001)

\section{INTRODUCTION}

Though raised by O'Hara for more than a decade ago, our understanding of uninformed traders' behavior and what traders can learn from market information is still very limited, in particular in limit order markets, which are the dominant financial markets. Because of information asymmetry and growing complexity in order flows and trading in limit order markets, the current literature mainly focuses on the behavior of informed traders and simplifies the behavior of uninformed traders by introducing private value (Goettler, Parlour and Rajan (2009)) or time preference (Rosu (2014)) to exogenously determine the trading strategies of uninformed traders.

In this paper, we focus on the behavior heterogeneity between informed and uninformed traders when they share the same learning algorithms. Different from informed traders, uninformed traders have delayed information about the fundamental value. To examine how traders process market information, we introduce a genetic algorithm (henceforth GA) learning with a classifier system to classify market information into different groups according to market conditions. By combining information processing and order choice (limit/market orders to buy/sell) into the trading strategy, the behavior heterogeneity of traders is endogenously emerged from their learning and trading. Put differently, we allow trader to co-evolve trading strategies with gain information from short term price movement and, most importantly, the state of the order book itself. We show that, measured by the average usage of different group of market information, the trading strategies under the GA learning become stationary in long run. This helps us to understand the effect of learning on order profit, information efficiency, order submission and liquidity. We find that, with respect to information process, market information is more useful for uninformed traders than informed traders, in particular informed traders pay more attention to the last transaction sign while uninformed trader pay more attention to some technical rules. With respect to the impact on the market, learning of uninformed traders improves market information efficiency, which is not necessarily the case when informed traders learn. With respect to order submission, learning makes uninformed traders submit less aggressive limit orders but more market orders and patient limit orders, while it makes informed traders submit less market orders and more aggressive limit orders. The results provide some insight into the 
information processing and order submission behavior of traders when they learn from market information in limit order markets. They also lead to an implication on the probability of informed trading (PIN).

This paper contributes to the literature of market microstructure in limit order markets and agent-based computational finance with adaptive learning by integrating them into a unified framework to address the open question from O'Hara. In the literature of market microstructure in limit order markets, the endogenous order choice based on the order book conditions is a core and challenging issue, as highlighted by Rosu (2012) I In particular, modeling a dynamic limit order book is very challenging. As pointed out by Goettler et al. (2009) (p.68), "a model that incorporates the relevant frictions of limit-order markets (such as discrete prices, staggered trader arrivals, and asymmetric information) does not readily admit a closed-form solution." To overcome this challenge, we introduce a GA learning with classified rules into a limit order market. Within this framework, a trading strategy contains two parts: market conditions and actions. The market conditions may include market information of bid, ask, mid-price (the bid-ask midpoint), historical prices, order book depth and the transaction sign of last market order. Classified rules, such as "the current ask is lower than the last ask", are used for traders to process order book information. The actions may include buy/sell at market/limit orders. For example, one trading strategy could be: "when the current ask is lower than the last ask and the current bid-ask spread is smaller than before, traders choose a market buy". Trading strategies are then evolved according to the GA based on their historical performance. When a trader enters the market, he/she chooses the best strategies from the selected candidates that match the current market conditions. In this way, the GA allows traders to learn from their historical experience and endogenously make order choice according to the current market conditions. We show that, measured by the average usage frequency of different groups of information, trading strategies becomes stationary under the GA learning in long run, demonstrating the effectiveness of the learning. We also show that, with the GA learning, the average usage of all groups of information is higher for uninformed traders than for informed traders. In general, the order profit/loss of traders is improved/reduced and the learning is more valuable for uninformed traders than for informed traders. Among all the market information, informed traders use more information of the last transaction sign, while uninformed traders use more information related to technical rules. Furthermore, traders submit more limit orders than market orders and the learning reduces the bid-ask spread; however, the learning makes informed traders submit less market orders and more aggressive limit orders

\footnotetext{
1 Rosu (2014) solves the stationary equilibrium by assuming that traders can continuously monitor the market and reenter the market to modify their limit orders freely at anytime.
} 
while uninformed traders submit less aggressive limit orders but more market orders and limit orders at or away the quotes. Moreover, by using the information of the last transaction sign, informed traders improve their order profit, submit more market orders and hence consume more liquidity but reduce the bid-ask spread. By using the technical rules, uninformed traders reduce their loss, market information efficiency is improved but the bid-ask spread becomes wider. In a robustness analysis, we find that private value for uninformed traders can affect traders' trading strategies and limit order market significantly in short-run, but not in long-run. This result is different from Goettler et al. (2009) who find that private value mainly determines the order submission of uninformed traders. This result of endogenous order choice provides insight into how traders process order book information and how the learning affects the market liquidity through traders' order submission.

This paper also contributes to the literature of agent-based computational finance with adaptive learning.2 On the one hand, two surveys of Chen, Chang and Du (2012) and Gould, Porter, Williams, Fenn and Howison (2013) have pointed out that after several prototypes have successfully replicated a number of financial stylized facts of the low frequency data, the next milestone is to see whether the agentbased model can also be used to replicate the features either in the high frequency domain or the features in market microstructure, which may be considered as the two missions of the second-generation agent-based financial models. While there are some limited progresses made for the first mission 3 little progress has been made for the second. While there will be another long journey ahead in the second mission, this paper provides a framework within which many details (such as asymmetric information, learning and order choice) can be articulated. On the other hand, though adaptive learning has been widely used in financial markets, for example, a series papers based on the SFI-ASM (Arthur, Holland, LeBaron, Palmer and Tayler (1997)) and BH (Brock and Hommes (1998)) models. However, in most of these agent-based models with adaptive learning, the evolution of trading strategies is largely driven by the market price instead of the fundamental value, which is the focus of the microstructure literature in limit order markets. This difference from most of the agent-based financial models with adaptive learning brings a different dynamics 4 We find that the GA learning from uninformed traders improves market information efficiency, which is not necessarily the case when informed traders learn.

\footnotetext{
${ }^{2}$ We thank anonymous referees for suggestions of highlighting this aspect and the following discussions.

${ }^{3}$ See Chiarella, He, Shi and Wei (2014) and the references cited there for the latest development in this direction.

${ }^{4}$ In our model, large bubble and crash would not happen when the GA learning becomes effective, though market price can deviate from the fundamental value when the learning is driven by traders' order profits.
} 
This result is very interesting and new to the literature 5 The framework allows us to capture the effect of asymmetric information, which is the focus in the market microstructure literature since Kyle (1985) and to fill the gap between agent-based computational finance and mainstream market microstructure.

A robustness analysis on the fraction of informed traders leads to an interesting implication to the probability of informed trading (PIN). We find that the PIN is positively related to market volatility and the bid-ask spread, but negatively related to the ratio of market volatility to the bid-ask spread. This provides a useful implication for estimating the PIN in limit order markets.

Related literature - This paper is part of a growing literature in limit order market models with asymmetric information and agent-based models with adaptive learning. For limit order market models, some early static models of limit order markets assume that informed traders only use market orders and uninformed traders or liquidity traders only use limit orders (see, for example, Glosten (1994) and Seppi (1994)). Empirical studies find that both informed and uninformed traders use a mixture of market orders and limit orders 6 For more discussion about the choice of order type, we refer the reader to Parlour and Seppi (2008) and Rosu (2012). There are a few papers explain how the traders determine their order choice under asymmetric information. Menkhoff et al. (2010) empirically find that informed traders' order choice is sensitive to market conditions including the bid-ask spread, volatility, momentum of order flow and order book depth. Goettler et al. (2009) and Rosu (2014) introduce two dynamic models that allow informed and uninformed traders to determine their choice of order type in limit order markets. Goettler et al. (2009) assume that information is short-lived, so informed and uninformed traders choose buy or sell and order type by a trade-off among private value the expected order profit and pick-off risk. By assuming that uninformed traders have high private value while informed traders have zero private value, the trading strategies of the uninformed traders are mainly determined exogenously by the private value instead of asymmetric information. Rosu (2014) assumes that information is long-lived and traders' buy-sell decision and the time preference of order submission are exogenously determined. He finds that in equilibrium, the patient informed traders also

\footnotetext{
${ }^{5}$ Some models, such as Kluger and McBride (2011) and Anufriev, Arifovich, Ledyard and Panchenko (2013), find that adaptive learning improve market efficiency in limit order markets.

${ }^{6}$ See, for example, Keim and Madhavan (1995), Chakravarty and Holden (1995), Harris (1998), Bloomfield, O'Hara and Saar (2005), Kaniel and Liu (2006), Menkhoff, Osler and Schmeling (2010) and Collin-Dufresne and Fos (2013).

${ }^{7}$ According to Goettler, Parlour and Rajan (2005), the private value is not the private information, it reflects idiosyncratic motives for trade (such as wealth shocks, tax exposures, or hedging and portfolio rebalancing needs).
} 
submit market orders when the price deviates far from fundamental value. Therefore the order submission behaviors in these two models depend highly on exogenous parameters, such as private value and time preference. Due to analytical intractability, their models focus on stationary equilibrium and do not consider how traders learn from historical market information and do not allow traders to choose trading strategies endogenously based on the market conditions. Moreover, they assume that once the traders accomplish their transactions, they exit the market forever. This setup does not allow traders to learn from their trading experience. Empirically Nicolosi, Peng and Zhu (2009) find that traders learn from their trading experience, consequently adjust their behavior and effectively improve their investment performance. By introducing the GA learning with a classifier system, we allow traders to reenter the market to trade time after time and learn from their trading experience.

This paper uses the GA to model traders' adaptive learning and evolution of trading strategies 8 Since introduced first by Holland (1975), GA has been used in agent-based models to examine learning and evolution in financial markets. The GA is a search heuristic that mimics the process of natural selection. It generates solutions to optimization problems using techniques inspired by natural evolution, such as selection, crossover and mutation. In the initial stage, the GA randomly generates some solutions, which are evaluated by their performance. The GA then uses selection, crossover and mutation to evolve the solutions based on the survival of the fittest, and creates new solutions from solutions with better historical performance to replace under-performed solutions. In this way, the GA generates optimal sets of solutions to the changes in the environment. Early studies include Arifovic (1996), SFI-ASM (Arthur et al. (1997)) and Routledge (1999, 2001). Recently, to generate some intraday trading patterns in limit order markets, Kluger and McBride (2011) allow both informed and uninformed traders using a GA to decide when to enter the market during a trading day.

The classifier systems have been introduced in economic models, such as Marimon, McGrattan and Sargent (1990), Allen and Carroll (2001), Lettau and Uhlig (1999), and SFI-ASM models (such as Arthur et al. (1997), LeBaron, Arthur and Palmer (1999). More recently, Wei, Zhang, He and Zhang (2013) introduce a classifier systems to a GA learning model of the limit order book under a similar information structure to Goettler et al. (2009). It allows part of the uninformed traders to learn

\footnotetext{
${ }^{8}$ Other models with adaptive learning include Chakrabarti and Roll (2012) who use the Bayesian learning in a sequential game in a market for a single asset with fixed aggregate supply, and Pouget (2007) who employs experience weighted attraction learning in a call market and a Walrasian tatonnement market; both find that adaptive learning is useful for traders. In limit order book markets, LeBaron and Yamamoto (2008) employ GA to capture the imitation behaviour among heterogenous beliefs. Moreover, Darley and Outkin (2007) use adaptive learning to evolve trading strategies of market makers and apply their simulations to the Nasdaq market in 1998.
} 
and to forecast the current fundamental value by using GA. They show that the learning of uninformed traders improves information dissemination efficiency. More important, it provides a method on how to use a classifier system to describe the limit order market conditions, so that traders can optimize their forecasting rules based on market information. This paper extends the classifier system used in Wei et al. (2013) and introduces more detailed market conditions. As far as we know this is the first paper to apply classifier systems in a simulated microstructure environment trying to use it to convert states of the order book into order submission. 9

The rest of paper is organized as follows. Section 2 introduces the limit order market model and GA learning. Section 3 examines the evolution dynamics of trading strategies and analyzes the evolution of the usage of different groups of information. Section 4 focuses on the effect of learning on order profit, information efficiency, the bid-ask spread and order submission. Section 5 provides a robustness analysis on private value, slow evolution speed of GA and an implication to the PIN models. Section 6 concludes. A further robustness analysis on the fundamental volatility, the market share of informed traders, the information lag, and the weight on the recent profit in the performance measure of the GA are provided in the Appendix.

\section{THE ModeL}

We consider a continuous double auction limit order market model of trading a single risky asset. Traders are either informed or uninformed and their trading strategies are generated and updated endogenously through genetic algorithm learning as follows.

2.1. The limit order market. There are $N$ risk neutral traders including $N_{I}$ informed and $N_{U}$ uninformed traders with $N_{I}+N_{U}=N$. Each trader arrives at the market according to a Poisson process with parameter $\lambda$. The informed traders know the fundamental values when they arrive at the market. The information is short-lived, meaning that uninformed traders know the fundamental values with a time lag $\tau>0$, called information lag or information-lived time. There is a risky asset and a trading time period $t$, defined by $(t-1, t]$, corresponds to a short time interval such as one minute in the real market. The fundamental value $v_{t}$ of the risky asset follows a random walk process with an initial value of $v_{o}$. Innovations in the fundamental value $v_{t}$ occur according to a Poisson process with parameter $\phi$. When an innovation occurs, the fundamental value either increases or decreases by $\kappa$ tick sizes with equal probability. Depending on the value of the parameter

\footnotetext{
${ }^{9}$ We would like to thank an anonymous referee for pointing this out.
} 
$\phi$, innovations may occur several times in one time period (when $\phi>1$ ). All the informed traders who enter the market in time period $t$ know the (same) fundamental value $v_{t}$; however the uninformed traders know the fundamental value $v_{t-\tau}$ with $\tau>0$ measured in units of a time period 10 When entering the market, traders submit orders to buy or sell at most one unit of the asset 11 Transactions take place based on the standard price and time priorities in limit order markets.

When a trader enters the market at time $t^{\prime} \in(t-1, t]$ of time period $t$, he observes market information of history transaction prices and the current limit order book, including the best bid $b_{t^{\prime}}$ and ask $a_{t^{\prime}}$ prices 12 the mid-price $p_{t^{\prime}}^{m}=\left(a_{t^{\prime}}+b_{t^{\prime}}\right) / 2$, the spread $s_{t^{\prime}}=a_{t^{\prime}}-b_{t^{\prime}}$, the depths of the limit order book such as the depth at the best bid $d_{t^{\prime}}^{b}$ and the best ask $d_{t^{\prime}}^{a}$, the depth of the buy side $d_{t^{\prime}}^{b u y}$ and the sell side $d_{t^{\prime}}^{\text {sell }}$, and the average market price over the last $\tau$ periods defined by $\bar{p}_{t, \tau}=$ $\left[p_{t-1}+p_{t-2}+\cdots+p_{t-\tau}\right] / \tau$, here $p_{t-1}$ is the last transaction price in time $t-1$, and we let $p_{t}=p_{t-1}$ if there is no transaction between time $t-1$ and $t$. The trader also knows if the last transacted order was a buy or sell initiated transaction (denoted by $p_{t^{\prime}}^{ \pm},+$for a buy and - for a sell order). To reduce pick-off risk, the limit order expires in time $D$, which is set to be one trading day. To incorporate the learning, instead of assuming that traders can have at most one opportunity to trade as in the literature, we allow traders to reenter the market according to the Poisson process with parameter $\lambda$. Upon reentry, traders cancel their previous limit orders and submits new orders.

2.2. Trading strategies. Because of growing complexity of order flow, it is difficult, if not impossible, for traders in particular uninformed traders to find the best trading strategy to trade in order to make profit or reduce loss. To overcome this challenge, we introduce a genetic algorithm (GA) with a classifier system as a learning mechanism for traders to learn and trade.

When a trader arrives at the market, he uses a GA to learn from market information, plus the private information about the fundamental value if the trader is informed, and chooses the best trading strategies to buy or sell one share with either a market order or a limit order (to be specified later). The difference between

\footnotetext{
${ }^{10}$ The fundamental value and information structure follow Goettler et al. (2009).

11 As pointed out by Rosu (2012), most of limit order market models assumes risk-neutral traders with order size of one. To endogenize the order size decision and order-choice with asymmetric information is an important but complicated issue. This has been partially examined in Chiarella, Iori and Perellò (2009) with an exogenous order submission rule under symmetric information.

${ }^{12}$ Here $t^{\prime}$ refers to a trading time during the one minute time period. In some extreme case, with very small probability, either the buy or sell limit order book could be empty. To facilitate trading, we let $a_{t^{\prime}}=1.01 p_{t^{\prime \prime}}$ if the sell limit order book is empty and $b_{t^{\prime}}=0.99 p_{t^{\prime \prime}}$ if the buy limit order book is empty; here $t^{\prime \prime}$ denotes the previous time when the best quote is available.
} 
informed and uninformed traders is that the trading decisions for informed traders to buy or sell are determined by their private information of the fundamental value, but it becomes part of the learning for uninformed traders.

The learning mechanism of GA is based on the principles of natural selection. The outcome or solution of GA learning is called a chromosome, which is evaluated based on its historical performance and selectively evolved through processes of selection, crossover and mutation (to be explained in the latter half of this subsection). In the framework of Arthur et al. (1997), a classifier system is introduced so that an agent can recognize market conditions and choose the chromosome accordingly. Motivated by Arthur et al. (1997), we develop a classifier system as part of trading strategies to characterize market conditions and the limit order book. For each trader, a trading strategy $i$ contains two components. The first component corresponds to market conditions $x^{i}$ (say, for example, the current mid-price $p_{t^{\prime}}^{m}$ is higher than the expected fundamental value $v_{t}^{j}$ of trader $j$ ) and the second component is an action $y^{i}$ of buying or selling one unit of the stock at either a market or limit order. The trader then chooses the best trading strategy according to its strength mainly determined by its historical performance (to be specified later). We now provide some details about the two components of a trading strategy based on the GA learning.

As the first component, the market condition $x^{i}$ is characterized by classified rules (CRs) of the classifier system on the most important market information when traders make their decisions. There are a lot of information in limit order markets and the types of information we consider are mainly motivated by Goettler et al. (2009), Menkhoff et al. (2010) and Wei et al. (2013). Goettler et al. (2009) find that information about the change in ask/bid, the last transaction price, the last transaction sign (buy or sell), the depths at the quote and away from the quote significantly affects the expectation of the fundamental value for uninformed traders. Menkhoff et al. (2010) find that the order submission of informed traders is affected by the bid-ask spreads, volatility, momentum of order flow and order book depth. Chung, Gao, Lu and Mizrach (2013) find that the last transaction sign and the order book depth can significantly influence the short-term movement of return. The classifier system developed in the following extends the one introduced in Wei et al. (2013) who find that the forecasting accuracy of uninformed traders improves when they use the GA to learn from the lagged fundamental value, historical prices and the mid-price. In this paper, we consider information on the expected fundamental value, mid-price, historical prices and the order book information including recent change in quotes and the bid-ask spread, and order book depth imbalance and introduce ten CRs listed in Table 1 to classify market conditions.

The CRs in Table 1 are grouped based on five different types of market information. The first group, "Fundamental Value" (FV) contains the classified rule 
TABLE 1. The classified rules (CRs) based on expected fundamental value (Group FV, CR1), technical rules (Group TR, from CR2 to CR4), recent change in quotes and bid-ask spread (Group QS, from CR5 to CR7), order book depth imbalance (Group DI, CR8 and CR9), and the last transaction sign (Group TS, CR10). Here $v_{t}^{j}=v_{t}$ for informed traders and $v_{t}^{j}=v_{t-\tau}$ for uninformed traders.

\begin{tabular}{|c|c|c|c|}
\hline Group & Num & $\mathrm{CR}$ & Description \\
\hline $\mathrm{FV}$ & CR1 & $p_{t^{\prime}}^{m}>v_{t}^{j}$ & $\begin{array}{l}\text { The mid-price is higher than the expected fundamental } \\
\text { value. }\end{array}$ \\
\hline \multirow[t]{3}{*}{ TR } & $\mathrm{CR} 2$ & $\bar{p}_{t, \tau}>v_{t}^{j}$ & $\begin{array}{l}\text { The average market price of last } \tau \text { periods is higher than } \\
\text { the expected fundament value. }\end{array}$ \\
\hline & CR3 & $p_{t^{\prime}}^{m}>\bar{p}_{t, \tau}$ & $\begin{array}{l}\text { The mid-price is higher than the average market price of } \\
\text { last } \tau \text { periods. }\end{array}$ \\
\hline & $\mathrm{CR} 4$ & $\bar{p}_{t, \tau / 2}>\bar{p}_{t, \tau}$ & $\begin{array}{l}\text { The average market price of last } \tau / 2 \text { periods is higher } \\
\text { than the average market price of last } \tau \text {. }\end{array}$ \\
\hline \multirow[t]{3}{*}{$\mathrm{QS}$} & CR5 & $s_{t^{\prime}}>s_{t^{\prime \prime}}$ & The current spread is larger than the last spread. \\
\hline & CR6 & $a_{t^{\prime}}>a_{t^{\prime \prime}}$ & The current ask is higher than the last ask. \\
\hline & CR7 & $b_{t^{\prime}}>b_{t^{\prime \prime}}$ & The current bid is higher than the last bid. \\
\hline \multirow[t]{2}{*}{$\mathrm{DI}$} & CR8 & $d_{t^{\prime}}^{a}>d_{t^{\prime}}^{b}$ & $\begin{array}{l}\text { The current depth of the ask is larger than the current } \\
\text { depth at the bid. }\end{array}$ \\
\hline & CR9 & $d_{t^{\prime}}^{s e l l}>d_{t^{\prime}}^{b u y}$ & $\begin{array}{l}\text { The current depth of the sell side is larger than the } \\
\text { current depth of the buy side. }\end{array}$ \\
\hline TS & CR10 & $p_{t^{\prime \prime}}^{ \pm}$ & Last transaction sign (last market order is buy or sell). \\
\hline
\end{tabular}

CR1 describing the relations between the expected fundamental value and the midprice; the second group, "Technical Rules" TR, contains CR2 to CR4, describing the technical rules among the mid-price, the expected fundamental value and the average market prices of the last $\tau / 2$ and $\tau$ periods; the third group, "Quotes and Spread" (QS), contains CR5 to CR7 describing the recent change in the quotes and the bid-ask spread; the fourth group, "Depth Imbalance" (DI), contains CR8 to CR9, describing limit order book imbalance; and the last group, "Transaction Sign" (TS), contains CR10 describing the last transaction sign 13 We use binary strings to represent CRs and hence market conditions. For example, for CR1, "1" indicates that CR1 is true and "0" means that CR1 is false. Hence one binary string has 10 bits and every bit represents two states of each CR, for example, "101110 01101"

\footnotetext{
${ }^{13}$ If we let the agent consider all the information of the limit order book and prices of the past $\tau$ periods, the agent may learn better, but it leaves the set of classified rules too large and the learning more complicated. In our model, agents are bounded rational, and they can process part of information of the limit order book. In the classifier system, the average prices and the order book depth reflect part of information of past $\tau$ periods.
} 
indicates one possible market condition. Hence, there are totally $2^{10}=1024$ market conditions.

In principle, a trader needs at least 1,024 trading strategies to match all the market conditions. However, in some cases, some market information become irrelevant for traders, and in such case we use "\#" to replace 1 or 0 , indicating that the corresponding market information is not considered. Thus values of a classified rule in a trading strategy can be either 1,0 or \#, respectively indicating that the market condition is true, not true, or irrelevant. Hence a trading strategy with $n$ "\#" can match $2^{n}$ market conditions. For example, a conditional part of a trading strategy "\#0100 10001" can match both the market conditions "10100 10001" and "00100 10001". Therefore, each trader needs less than 1,024 strategies to match all market conditions. In addition, traders have limited learning ability to process all market information in every trade. To take this into account and to speed up the learning process, we assume that each trader only considers $2^{8}=256$ strategies.

The second component of a trading strategy is the action of trading. In general, a trader needs to choose from many types of orders. Goettler et al. (2005) classify orders into four types, including market order, aggressive limit order, limit order at the quote, and limit order away from the quote. Similarly, in Menkhoff et al. (2010) orders are classified into market orders, aggressive limit orders, and patient limit orders (limit orders at the quote and limit orders away from the quote). In this paper, we follow Goettler et al. (2005) and classify orders into four types: a market order $(M O)$, a limit order at the quote $(L O A)$, an aggressive limit order $(A L O)$, and an unaggressive limit order ( $U L O$, or limit order away from the quote). To simplify the analysis, we define an aggressive limit order $(A L O)$ to be the limit order above the bid or below the ask by one tick, and an unaggressive limit order $(U L O)$ to be the limit order below the bid or above the ask by one tick 14 Therefore an $A L O$ narrows the bid-ask spread and improves the liquidity, while a $L O A$ does not narrow the bid-ask spread but supplies immediate liquidity. Given the two sides of the book and the four types of orders, there are 8 actions in total, listed in Table 2. We use three binary bits to describe actions. For example "000" means a market buy $(M B)$ order.

TABLE 2. The actions or order types of trading strategies

\begin{tabular}{ccc|ccc}
\hline Action (buy) & Binary code & Description & Action(sell) & Binary code & Description \\
\hline MB & 000 & Market buy & MS & 111 & Market sell \\
ALB & 001 & Aggressive limit buy & ALS & 110 & Aggressive limit sell \\
LBB & 010 & Limit buy at the bid & LSA & 101 & Limit sell at the ask \\
ULB & 010 & Unaggressive limit buy & ULS & 100 & Unaggressive limit sell \\
\hline
\end{tabular}

\footnotetext{
${ }^{14}$ Generally, the $A L O$ or $U L O$ can deviate from the quote in several ticks. To simplify the analysis, we set the deviation as one tick.
} 
By combining the two components, a trading strategy $\left(x^{i}, y^{i}\right)$ for a trader means to take an action $y^{i}$ under market condition $x^{i}$. For example, one possible trading strategy $i$ is defined when $x^{i}$ is given by " $1 \# 1 \# 100 \# 1 \#$ " and $y^{i}$ is given by " 000 " 15 For an informed trader, since he knows the fundamental value, his buy/sell decision is determined by comparing the fundamental value to the bid and ask, and he uses GA to optimize his order aggressiveness. For an uninformed trader, he uses the GA to optimize both the buy-sell decision and order aggressiveness.

2.3. The GA Learning. We consider individual GA learning, meaning that each trader constructs his own set of trading strategies using the GA. The evolution process of the GA includes selection, crossover and mutation. In the selection process, a trading strategy is selected by a tournament mechanism based on its strength $\eta^{i}=\pi^{i}-\delta^{i}$, where $\pi^{i}$ represents the performance and $\delta^{i}$ measures the specificity of a trading strategy 16 The performance $\pi^{i}$ of trading strategy $i$ for a trader when he enters the market at time $t^{\prime}$ is updated (with an initial value of zero) according to

$$
\pi_{t^{\prime}}^{i}=\pi_{z}^{i}=\beta r_{z}^{i}+(1-\beta) \pi_{z^{\prime}}^{i}
$$

where $\beta \in[0,1]$ and $r_{z}^{i}$ is the order profit of strategy $i$ with $r_{z}^{i}=v_{z}-p_{z}$ for an executed buy order, $r_{z}^{i}=p_{z}-v_{z}$ for an executed sell order, or $r_{z}^{i}=0$ for an canceled or expired order at the last trading time $z$. This means that the performance $\pi^{i}$ of trading strategy $i$ for the trader is a weighted average of his recent order profit $r_{z}^{i}$ and his previous performance $\pi_{z^{\prime}}^{i}$ of the strategy. A large $\beta$ means that the trader weights more on the recent profit and less on the historical performance of the strategy.

When a trader enters the market initially, all his trading strategies are randomly generated with most "\#" bits in the market condition part 17 With an initial performance of zero, the strength of a trading strategy for the trader is low. When a submitted order has been executed, or canceled, or expired, the performance and

\footnotetext{
${ }^{15}$ In some special limit order book scenarios, certain types of actions or orders are impossible or unused. For example, when the buy-side of the limit order book is empty, traders can not submit a market sell $M S$. These special scenarios are listed in Table 11 of the Appendix.

${ }^{16}$ The specificity measures the fitness or cost of a trading strategy. For example, for a trading strategy with "1\#1\#1 00\#1\#", the number of specific bits (non-omitted bits) $m$ is equal to 5 . The specificity of a trading strategy is equal to $m \mu$, where $\mu$ is the bit cost, a small value such as 0.001. Hence, if two trading strategies have the same performance $\pi^{i}$, the trading strategy with less specificity (with more omitted bits, more adaptability) has a higher strength.

${ }^{17}$ Because the GA needs to use the historical data of the last $\tau$ periods to generate technical rues, we let the market "warm up" for $\tau$ periods before traders use the GA to trade. During the period, traders randomly submit orders.
} 
Crossover

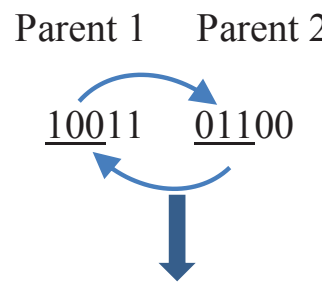

Child 1: $\underline{01111} \&$ Child 2: 10000

\section{Mutation}

\section{Parent 1}

$1 \underline{0} 011$

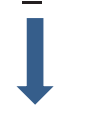

Child: $1 \underline{1011}$ or $1 \underline{\# 011}$

Figure 1. The crossover and mutation processes of a genetic algorithm.

hence the strength of the strategy are updated by the trader 18 The trader ranks the performance of all his trading strategies based on the strengths and selects the top $10 \%$ of his strategies to generate new strategies and replaces the bottom $10 \%$ of his strategies. The new strategies are generated through the processes of crossover and mutation according to given probabilities. Crossover means that, with a certain probability called the crossover rate, the trader randomly chooses two trading strategies from the top $10 \%$ of the strategies as parents, splits each trading strategy into two parts at a random bit and then swaps the two parts to create two new trading strategies as children. This process is illustrated in Figure 1, For example, the two parents trading strategies are "10011" and "01100" 19 If they are split at the third bit, then the two new child strategies are "01111" and "10000". Mutation means that, with a certain probability called the mutation rate, the trader randomly selects a high strength trading strategy as a parent and makes a random bit change of the parent trading strategy to a different value. As illustrated in Figure 1 for the parent trading strategy "10011", the second bit is chosen to mutate, then the child strategy becomes either "11011" or "1\#011". When the market condition parts cross (mutate), the action parts also cross (mutate) with the same crossover (mutation) probability. The strength of the child trading strategy is equal to the average strength of the parents under crossover and the strength of the parent under mutation (minus its specificity $\delta^{i}$ ).

2.4. A benchmark model. To examine different learning effect of informed and uninformed traders, we consider a benchmark model (BM) in which both informed

\footnotetext{
${ }^{18}$ For the informed trader, the performances of his strategies are updated immediately when he enters the market. For the uninformed traders, due to the information lag, the performances of his trading strategies are updated only when the transactions occur before or at period $t-\tau$.

${ }^{19}$ In our GA with the classifier system, the condition part is a 10-bits string and the act part is a 3-bits string. To illustrate, we use 5-bits strings as an example.
} 
and uninformed traders use the GA to learn and compare to the models in which only informed or uninformed traders learn. For convenience of the discussion, we refer the benchmark model to a two-side learning model and the other models to one-side learning models.

For the BM model, we choose an initial fundamental value of $v_{0}=20$, initial market price $p_{0}=v_{0}=20$, the tick size 0.01 , and the total number of traders in the market to be 1,000. Based on some empirical studies on the probability of informed trading (PIN) 20 we set the proportion of the informed traders to be $10 \%$ in the BM, which corresponds to 100 informed traders and 900 uninformed traders. On the volatility of the fundamental value, we choose the Poisson rate $\phi=1$ and $\kappa=421$ This implies that, on average, the innovation of the fundamental value occurs once every minute and each innovation changes the fundamental value by four ticks.22 For the information lag, we set $\tau=360$. Considering that one period corresponds to one minute, $\tau=360$ implies that the time-lag of the fundamental values is 6 hours, which is one trading day in the Australian Stock Market. We choose the maximum order survival time as one trading day $D=360$. Traders (both informed and uninformed) arrive the market following a Poisson process with an arrival rate of $\lambda=1 / 60$, meaning that each trader enters the market once per hour or 6 times per day on average 23 We set the discount rate of historical performance $\beta=0.2$, the crossover rate to be 0.1 and the mutation rate to be 0.3 . In Section 5 , we conduct a robustness analysis by considering shorter information lag of $\tau=180$ and a smaller $\beta=0.05$. To achieve statistical significance we run 30 simulations. The evolution process of a GA is active on average of 360 periods, one trading day, which is called one generation 24 To make the learning more efficient, each simulation runs 180,000 periods, so the evolution process is active for 500 times or generations.

\footnotetext{
${ }^{20}$ The PIN models in the literature, such as Yan and Zhang (2012) find that the PIN is between $10 \%$ to $20 \%$ on average. Since informed traders may have more transactions than uninformed traders, the proportion of informed traders may be less than the PIN level. Therefore we set the proportion of the informed traders to be $10 \%$ in the BM case. We also consider the fraction of informed traders to $5 \%$ and $90 \%$ in a robustness test later.

${ }^{21}$ Based on the empirical studies in the Australian Stock Market, the standard deviation of 1-minute mid-price returns is about 17 basic points of all stocks of ASX (Comerton-Forde and Putniṇš (2013)) and about 13 basic points of ASX200 (Putniṇš and Michayluk (2013)). Given the price level of 20, the average change of price in tick size is about 3.4 tick for all the ASX stocks and 2.6 ticks for the ASX200 stocks. We also conduct a robustness test for a lower volatility of $\kappa=2$ later.

${ }^{22}$ Following this innovation process, the fundamental value may be negative with a very small probability. So we set the minimum fundamental value to 5 to avoid a negative state.

${ }^{23}$ Due to limited attention, traders do not continuously monitor the market (Dugast (2012)).

${ }^{24} \mathrm{We}$ also conduct a robustness test for a slower evolution speed of 720 and 1,800 periods.
} 
To examine the impact of learning on the limit order market, including market efficiency and traders' order submission behavior, we need to understand whether the GA learning becomes stationary and, if so, what kind of information traders use when making decision. In particular, we are interested in how market information is used differently by informed and uninformed traders. To measure the stationarity of the learning, we introduce in the next section the average usage, or using frequency, of market information defined by the ten classified rules and examine the evolution of trading strategies.

\section{The EVOlution of TRADing STRATEGIES}

In this section, we consider the evolution of trading strategies and examine how the GA learning helps traders to process market information for the benchmark model (BM). To examine what kind of market information traders use to trade, we introduce usage frequency $\gamma_{i} \in[0,1]$ of the classified rule (CR) group $i$ defined in the Section 2.2. The five CR groups are "Fundamental Value" (FV), "Technical Rules" (TR), "Quotes and Spread" (QS), "Depth Imbalance" (DI) and "Transaction Sign" (TS), respectively. When a trader selects a trading strategy to trade, we look at the condition part of the strategy and count the bits in the corresponding classified rule which are not "\#" 25 For example, if the condition part of a trading strategy is represented by "\#\#1\#1 00\#1\#", the classified rules CR1, CR2, CR4, CR8 and CR10 with "\#" are not used and therefore not counted; while CR3, CR5, CR6, CR7 and CR9 are counted for one time. In this way, we calculate the total counts for each classified rule for informed and uninformed traders respectively. The average usage frequency $\gamma_{i}$ of the classified rule group $i$ for the informed (uninformed) traders is calculated by the ratio of the averaged total counts of the classified rules in group $i$ to the total trading times of informed (uninformed) traders in one generation. The total trading times of informed (uninformed) traders is equal to the number of periods for one generation (360 periods), multiplies by the arrival rate $\lambda=\frac{1}{60}$, and then multiplies by the number of informed (uninformed) traders $N_{I}=100$ $\left(N_{U}=900\right)$. The average usage frequency thus characterizes the usage of different types of information under the classified rules. Put differently, it represents the average probability of the usage of every classified rule per trade. For example, for CR1 to CR4, if the total counts of informed traders in one generation are 210, 220, 180 and 240, respectively, then the average usage frequency of the classified rule

\footnotetext{
${ }^{25}$ It would be interesting but challenging to examine both parts of the trading strategy and find out explicitly what market conditions trigger different types of orders; we leave this to the future research.
} 
groups FV and TR for the informed traders is defined by, respectively,

$$
\begin{gathered}
\gamma_{F V}=\gamma_{C R 1}=\frac{210}{360 * \frac{1}{60} * 100}=0.350 \\
\gamma_{T R}=\frac{1}{3}\left(\gamma_{C R 2}+\gamma_{C R 3}+\gamma_{C R 4}\right)=\frac{\frac{1}{3}(220+180+240)}{360 * \frac{1}{60} * 100} \approx 0.356 .
\end{gathered}
$$

This implies that on average the informed traders use the fundamental value information (FV) with a probability of 0.35 and the information based on the technical rules (TR) with a probability of 0.356 per trade. Examining the evolution of the usage frequency over generations helps to understand how different group of market information is used under the GA and wether the learning process of the trading strategy becomes stationary.

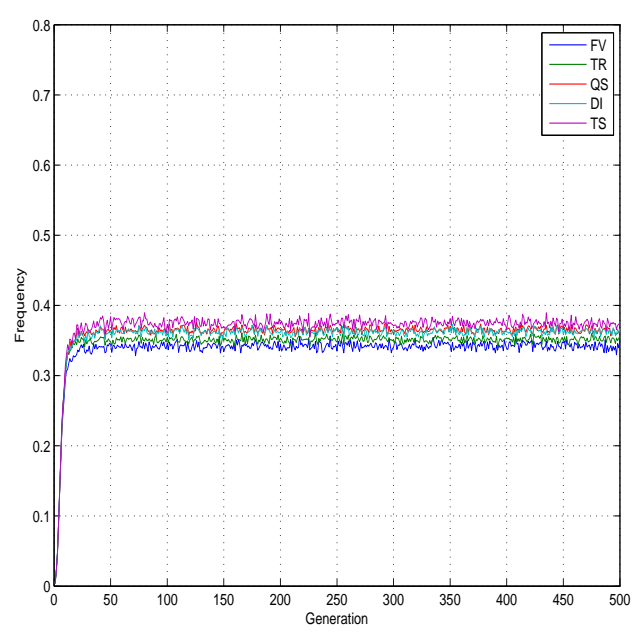

(a) Informed traders

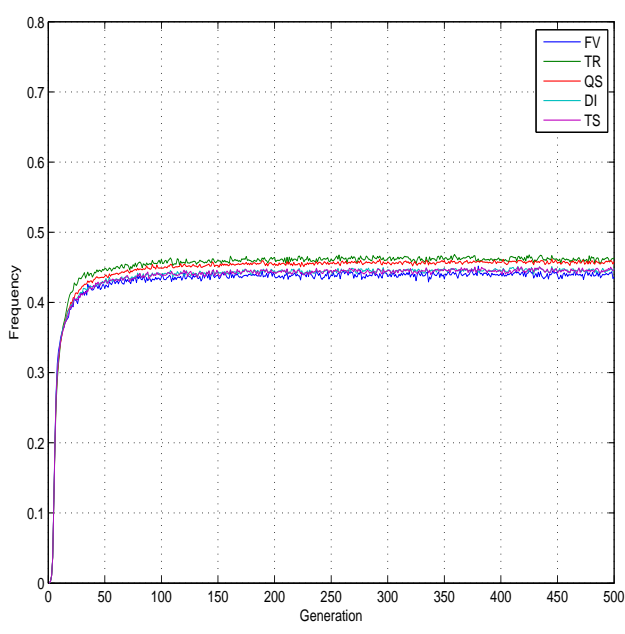

(b) Uninformed traders

Figure 2. The average usage frequency per trade $\gamma_{i}$ of each classified rule group $i$ for informed traders (a) and uninformed traders (b) in 500 generations (trading days). Different colours represent different CR groups.

If the GA learning is effective, more market information will be incorporated into the trading strategies and hence the average usage of the classified rules is expected to increase over the initial generations. In Fig. 2 we report the average usage frequencies of each classified rule group over 500 generations for informed (a) and uninformed (b) traders. The results lead to two observations. First, all the $\gamma_{i}$ for both informed and uninformed traders increase quickly in the early generations and then are stabilized with small fluctuates around some mean levels. Also the speed of the convergence is faster for informed than uninformed traders, taking about 50 generations for informed traders (in Fig. 2 (a)) and 200 generations for uninformed traders (in Fig. 2 (b)). Secondly, the average usage frequency for 
uninformed traders is higher than for informed traders. Fig. 2 shows that the average usage across different $\mathrm{CR}$ groups settles down to about 0.35 for informed traders and 0.45 for uninformed traders. This means that on average each group of information is used with probability of 0.35 for the informed traders and 0.45 for the uninformed traders in each trading strategy. Intuitively, compare to uninformed traders, informed traders do not need to use the GA learning to make the buy-sell decision and therefore their average usage of market information is lower.

Based on the first observation, the average usage frequencies across all CR groups seem to become stationary in long run. To verify this, we first estimate the distribution of $\gamma_{i}$ over the last 200 generations (from the generation 301 to the generation 500) across all CR groups. It is found that the distribution of $\gamma_{i}$ across different CR group $i$ approximates to a normal distribution. We then split the series of $\gamma_{i}$ into two parts with 100 generations in each part and find that the first half has the same mean as the second half based on ANOVA (Analysis of Variance). Therefore we conclude that, measured by the average usage frequency, the learning process becomes stationary in long run. It is the stationarity property of the learning that enables us to examine the impact of the learning on the market and traders' order submission, to be explored in the following section.

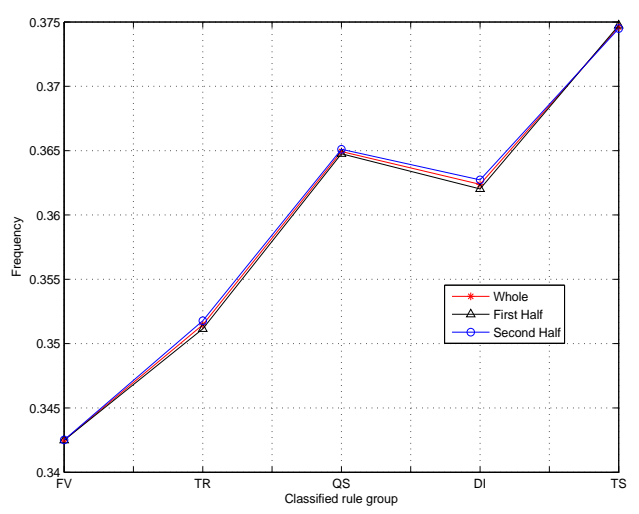

(a) Informed traders

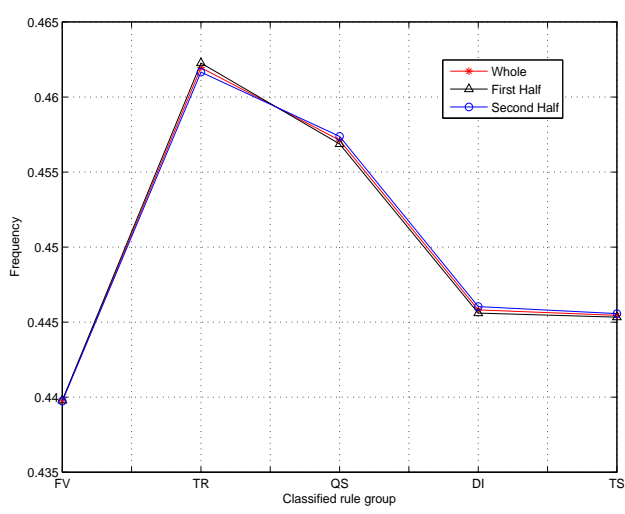

(b) Uninformed traders

Figure 3. The patterns of the means of the whole series, the first half and the second half of $\gamma_{i}$ among different classified rule groups of informed traders (a) and uninformed traders (b).

We further examine whether the patterns of $\gamma_{i}$ among different CR groups are the same. We report the patterns in Fig. 3 for for informed traders in (a) and uninformed traders in (b). Apart from showing that the average usage of information is higher for uninformed than for informed traders, the average usage frequency under the classified rules varies from 0.343 to 0.375 for informed traders and 0.440 to 0.462 for uninformed traders (indicated by the mean values in Table 12 in Appendix 
B). Furthermore, among different groups of information, the average usage of the CR group TS related to the last transaction sign is the highest for the informed traders, while for the uninformed traders, the highest is the CR group TR related to comparison of the moving average prices to the expected fundamental value and the mid-price 26 This result clearly indicates that, because of information asymmetry, the informed traders use more information on the last transaction while the uninformed traders use more information on technical rules, in particular moving average 27 when they submit their orders. In the next section, we further explore the impact of such difference between informed and uninformed traders on order profit, market information efficiency and order submission. It is interesting to note that market information of moving averages is useful for not only the uninformed traders, but also the informed traders.

The results above demonstrate that the GA learning becomes stationary in longrun. In a double auction market, Arthur et al. (1997) find that the GA with a classifier system makes the market price converge to rationally expected prices. To our knowledge, this is the first paper showing the stationarity of the GA learning with a classifier system in a limit order market. In a limit order market model, Goettler et al. (2009) employ a Markov perfect Bayesian equilibrium, which is stationary and symmetric and each type of traders can choose the same strategy. Traders' strategies do not depend on market conditions when they enter the market. This is different from our model in which traders' strategies depend on market conditions and information asymmetry. The stationarity of the GA learning can be used to characterize stationary trading strategies in limit order markets. More importantly, this allows us to provide insight into how informed and uninformed traders use the order book information to endogenously determine their order choice, which is a key issue in limit order markets highlighted in a survey of Rosu (2012).

\section{The LEARning EFFECT}

With the stationary GA learning shown in the previous section, we are able to examine the effect of the learning on the order profit, information efficiency, the bid-ask spread and order submission. We also examine the effect by comparing the two-side learning of the BM model to the one-side learning models in which only the uninformed or informed traders learn, respectively. To explore the role of the information process, we then focus on the effect of the most used classified rules

\footnotetext{
${ }^{26}$ We also use ANOVA and show that this finding is significant, illustrated by Fig. 8 8 in Appendix C.

${ }^{27}$ Since Group TR includes three CRs, we use ANOVA to analyze all the CRs and report the result in Fig. 9 of Appendix C. It shows that the average usage of CR4, the moving average rules of market price, is the highest.
} 
obtained in the previous section. Since the GA learning becomes stationary after 300 trading days, we take the last 200 trading days (the last 36,000 periods) for the analysis, and we set the total number of the analysis periods $T=36,000$.

We first introduce measures for order profit and information efficiency and notations on order submission. The order profit per trade $r_{t}$ is calculated by the difference between the transaction price $p_{t}$ of the executed order and the fundamental value $v_{t}$ and $r_{t}=v_{t}-p_{t}$ for an executed buy order and $r_{t}=p_{t}-v_{t}$ for an executed sell order. We denote $r_{I}$ and $r_{U}$ as the average order profit per trade of the informed and uninformed traders, respectively. Note that our model is a zero-sum game. Because of the information advantage, the order profit is positive for informed traders and negative for uninformed traders. For market information efficiency, we follow Theissen (2000) and use Mean Relative Error (MRE) to measure the relative error of the market price from the fundamental value,

$$
M R E=\frac{1}{T} \sum_{t=1}^{T} \frac{\left|p_{t}-v_{t}\right|}{v_{t}} .
$$

For order submission, we classify orders of type $j$ traders $(j=I$ for the informed traders and $j=U$ for the uninformed traders) into four classes: market buy/sell orders $M B_{j} / M S_{j}$, unaggressive limit buy/sell orders $U L B_{j} / U L S_{j}$, limit buy/sell orders at quote $L B A_{j} / L S A_{j}$, and aggressive limit buy/sell orders $A L B_{j} / A L S_{j}$. We denote

$$
\begin{gathered}
M O_{j}=M B_{j}+M S_{j}, \quad U L O_{j}=U L B_{j}+U L S_{j}, \\
L O A_{j}=L B A_{j}+L S A_{j}, \quad A L O_{j}=A L B_{j}+A L S_{j}
\end{gathered}
$$

the aggregate orders of the four classes of orders.

For the two-side learning of the BM model and one-side uninformed learning (UL) and informed learning (IL) models, we report the order profit, information efficiency and the bid-ask spread in Table 3. We also report the average number of different orders per trader over 600 trades in Table 4 , together with the executed limit orders $E L O_{I} / E L O_{U}$ for the informed in the upper panel and uninformed traders in the lower panel.

\subsection{The effect of learning on order profit, information efficiency, the bid-} ask spread and order submission. For the BM two-side learning model, the results in Table 3 show an average order profit of 35.19 ticks for the informed trader and -3.51 ticks for the uninformed trader per trade. The information efficiency $M R E$ is $2.21 \%$ and the bid-ask spread is 6.24 ticks. On order submission, the results for the BM model in Table 4 show that both informed and uninformed traders place more limit orders (all together) than market orders. Also, the informed traders 
TABLE 3. The learning effect on order profit $r_{I}$ and $r_{U}$ in ticks, information efficiency $M R E$ and the bid-ask spread $s$ in ticks. The value in bracket is the standard deviation of the 30 simulations, and $*$ indicates the significant difference between the BM model and the other model (under ANOVA with $p$-value for the relevant $F$ - statistic is less than $1 \%$, (the following tables are the same), similarly hereinafter in later tables.

\begin{tabular}{ccccc}
\hline Case & $r_{I}$ & $r_{U}$ & $M R E$ & $s$ \\
\hline BM & 35.19 & -3.51 & $2.21 \%$ & 6.24 \\
& {$[4.61]$} & {$[0.45]$} & {$[1.37 \%]$} & {$[2.91]$} \\
UL & $31.74^{*}$ & $-3.23^{*}$ & $1.49 \% *$ & $7.28^{*}$ \\
& {$[4.69]$} & {$[0.46]$} & {$[0.69 \%]$} & {$[3.58]$} \\
IL & $59.84^{*}$ & $-8.37^{*}$ & $4.06 \%^{*}$ & $16.00^{*}$ \\
& {$[4.49]$} & {$[0.59]$} & {$[1.90 \%]$} & {$[7.71]$} \\
\hline
\end{tabular}

TABLE 4. The learning effect on order submission.

\begin{tabular}{c|ccccc}
\hline Case & $M O_{I}$ & $A L O_{I}$ & $L O A_{I}$ & $U L O_{I}$ & $E L O_{I}$ \\
\hline $\mathrm{BM}$ & 155 & 100 & 172 & 173 & 149 \\
& {$[1]$} & {$[2]$} & {$[2]$} & {$[2]$} & {$[2]$} \\
$\mathrm{UL}$ & $168^{*}$ & $97^{*}$ & $168^{*}$ & $168^{*}$ & $143^{*}$ \\
& {$[1]$} & {$[3]$} & {$[1]$} & {$[2]$} & {$[1]$} \\
$\mathrm{IL}$ & $147^{*}$ & $133^{*}$ & $161^{*}$ & $159^{*}$ & $227^{*}$ \\
& {$[1]$} & {$[3]$} & {$[2]$} & {$[1]$} & {$[2]$} \\
\hline Case & $M O_{U}$ & $A L O_{U}$ & $L O A_{U}$ & $U L O_{U}$ & $E L O_{U}$ \\
\hline BM & 169 & 96 & 167 & 168 & 170 \\
& {$[1]$} & {$[3]$} & {$[1]$} & {$[1]$} & {$[1]$} \\
$\mathrm{UL}$ & $168^{*}$ & $97^{*}$ & 167 & 168 & 171 \\
& {$[1]$} & {$[2]$} & {$[1]$} & {$[1]$} & {$[1]$} \\
$\mathrm{IL}$ & $153^{*}$ & $133^{*}$ & $157^{*}$ & $157^{*}$ & $144^{*}$ \\
& {$[1]$} & {$[3]$} & {$[2]$} & {$[1]$} & {$[1]$} \\
\hline
\end{tabular}

submit less market orders and more limit orders (of all types) than the uninformed traders, which is consistent with Goettler et al. (2009).

To examine the effect of learning, we compare the results of two-side learning model to the one-side learning models. We first study the effect of learning from the informed traders by comparing the results for the UL model (in which only the uninformed traders learn) to the BM model (in which both informed and uninformed learn). Table 3 shows that, when the informed traders learn, the order 
profit increases by about 10\% (from 31.74 to 35.19 ticks) for the informed traders and decreases by about $10 \%$ (from -3.23 to -3.51 ticks) for the uninformed traders. If we decompose the total order profit of the informed traders in the BM into the profits generated by their private information and learning respectively, the results show that about $90 \%(=31.74 / 35.19)$ of their profit is from their private information and $10 \%(=(35.19-31.74) / 35.19)$ is from the learning. This implies that, informed traders' profit is mainly from their information advantage, but the learning also helps to increase their order profit. Interestingly, the $M R E$ increases by $0.72 \%$ (from $1.49 \%$ to $2.21 \%$ ) while the bid-ask spread is reduced by about one $(\approx 7.28-6.24)$ tick. This implies that the learning from the informed traders reduces market information efficiency but increases market liquidity. To provide an explanation, we examine the order submission behavior of traders. Table 4 shows that, when the informed traders learn, their market orders $M O_{I}$ decrease by about $8 \%$ (from 168 to 155 ) but the limit orders increase by about $3 \%$, in particular, the executed limit orders increase by more than $4 \%$. However, there is no significant change in order submission for the uninformed traders. This indicates that the reduction in the market information efficiency may be due to the manipulation of the informed traders by setting the prices through submitting more limit orders and less market orders, while the reduction in the bid-ask spread is due to the increase in liquidity supply and the decrease in liquidity consumption.

Next we examine the effect of learning from the uninformed traders by comparing the results of the IL model (in which only the informed traders learn) to the BM model. Table 3 shows that, when the uninformed traders learn, the order profit for the informed traders decreases by about $41 \%$ (from 59.84 to 35.19 ticks) and the loss for the uninformed traders reduces by about $58 \%$ (from -8.37 to -3.51 ticks). Also, the learning from the uninformed traders significantly reduces $M R E$ by $1.85 \%$ (from $4.06 \%$ to $2.21 \%)$ and the spread by about $10(\approx 16-6.24)$ ticks. For order submission, Table 4 shows that both the informed and uninformed traders increase their market orders and limit orders at or away from the quote (by about 5\% to $15 \%$ ) but reduce their aggressive limit orders significantly (by about 25\% for the informed traders and $28 \%$ for the uninformed traders). Note that the executed limit orders reduce by about $34 \%$ for the informed traders but increase by about $19 \%$ for the uninformed traders. As the result, both information efficiency and market liquidity are improved.

In summary, comparing to the learning from the informed traders, the learning from the uninformed traders has more significant impact on the order profit. It reduces the profit per trade of the informed traders (by about 41\%) and the loss per trade of the uninformed traders (by about 58\%). It makes the traders submit more market orders and limit orders at or away from the quote but less aggressive limit 
orders. It improves market information efficiency and liquidity. Some literature on GA learning, such as Kluger and McBride (2011) and Anufriev et al. (2013) shows that learning is effective and helpful for market efficiency. Our results show that the learning of uninformed traders improves the market information efficiency; however, informed traders may learn to manipulate the market which in turn reduce market information efficiency. This result is interesting and new to literature. More importantly, apart from a few studies ${ }^{28}$ this is the first paper that provides some insight into how learning can affect order submission differently for informed and uninformed traders.

4.2. The effect of the classified information. We have shown in the previous section that, when traders make their decisions by the GA learning, the usefulness of different types of information characterized by the classified rules can be different for informed and uninformed traders. The average usage of all types of information is high (about 45\%) for the uninformed traders and low (about 35\%) for the informed traders. We further show that the information of the last transaction sign under the classified rule group TS is the most used, about $37.5 \%$ for the informed traders, while for the uninformed traders, it is the technical rule group TR, about $46.2 \%$. It is important, but also challenging, to understand how different types of information affect traders' order submission and market efficiency. To tackle this challenge, we focus on the most used information (instead of all types of information) to illustrate the information effect. We consider two cases, one is denoted as NTS in which the classified rule group TS is removed for the informed traders only, and the other is denoted as NTR in which the classified rule group TR is removed for the uninformed traders only 29 By comparing NTS to the BM case, we examine the effect of the information of the last transaction sign for the informed traders. Similarly, by comparing NTR to the BM case, we examine the effect of the technical rules for the uninformed traders. The results on the effect of order profit and market efficiency are reported in Table 5 .

\footnotetext{
${ }^{28}$ In an empirical study, Nicolosi et al. (2009) find that individual traders do learn from their trading experience to get better forecasting of signal precision, and consequently adjust their portfolio management behavior and thus efficiently improve their profit and order submission. In another empirical study, Grinblatt, Keloharju and Linnainma (2012) find that high IQ traders submit more market orders when the bid-ask spreads temporarily narrow and have better executions than low IQ traders. They conjecture that high IQ traders are more capable of exploiting and processing limit order market information.

${ }^{29}$ Note that the classified rule group TS stays for the uninformed traders in the NTS case and the classified rule group TR stays for the informed traders in the NTR case; the other parameters of the two cases remain the same as in the BM case.
} 
TABle 5. The effects of the Group TS of informed trades and the Group TR of uninformed traders.

\begin{tabular}{ccccc}
\hline Case & $r_{I}$ & $r_{U}$ & $M R E$ & $s$ \\
\hline BM & 35.19 & -3.51 & $2.21 \%$ & 6.24 \\
& {$[4.61]$} & {$[0.45]$} & {$[1.37 \%]$} & {$[2.91]$} \\
NTS & $34.45^{*}$ & $-3.41^{*}$ & $1.83 \% *$ & $7.54^{*}$ \\
& {$[4.06]$} & {$[0.42]$} & {$[1.37 \%]$} & {$[4.06]$} \\
NTR & $38.16^{*}$ & $-3.82^{*}$ & $3.52 \% *$ & $4.89^{*}$ \\
& {$[4.39]$} & {$[0.43]$} & {$[1.70 \%]$} & {$[3.83]$} \\
\hline
\end{tabular}

We first compare NTS to BM. Table 5 shows that, by considering the information on the last transaction signs (group TS), the average order profit increases by about $2 \%$ (from 34.45 to 35.19 ticks) for the informed traders but decreases by about $2 \%$ (from -3.41 to -3.51 ticks) for the uninformed traders. Also, the market efficiency is reduced, but the spread becomes narrow. This result is consistent with the learning effect of the informed traders we discussed in the previous subsection. For the order submission, the results in Table 13 of Appendix D show that the informed traders submit more market orders (by about 2.5\%) and slightly less limit orders, while there is no significant change for the uninformed traders. Therefore, with the information of the last transaction signs, the informed traders improve their order profit and consume liquidity. The decrease in the information efficiency also reflects that the last transaction sign may help informed traders to manipulate the order book. The bid-ask spread also reduces because the informed traders learn better with the last transaction sign.

Next we compare NTR to BM. Table 5 shows that, by using the technical rules (group TR), the average order loss for the uninformed traders is reduced by about $8 \%$ (from -3.82 to -3.51 ticks), while the profit for the informed traders is also reduced by about $8 \%$ (from 38.16 to 35.19 ticks). The market efficiency is improved but the spread becomes wider. For the order submission, the results in Table 13 of Appendix D show that there is no significant change in order submission except the uninformed traders submit slightly more aggressive limit orders and less patient orders, which is consistent with Menkhoff et al. (2010) who show that uninformed traders treat aggressive limit order as a substitute for patient limit order. Note that the number of the executed limit orders is higher for the uninformed traders than for the informed traders. Therefore, using the technical rules, the uninformed traders can improve their order profit significantly. It seems that using of technical rules may increase the spread, but helps the uninformed traders to submitting more 
corrected orders, in particular the aggressive limit orders. This result is consistent with the broad use and success of momentum like strategies in finance 30

In summary, we show that learning and information are valuable for all traders, though the role of learning based on different types of information can be different for informed and uninformed traders. Because of the information disadvantage, learning becomes more valuable for uninformed traders. The analysis in this section provides an insight into how learning and different types of information affect the order profit, order submission, market liquidity and information efficiency differently.

\section{ROBUSTNESS ANALYSIS AND IMPLICATIONS}

The previous section focuses on the learning and classified information effect and the results are mainly based on the BM model. In this section, we conduct a robustness analysis to examine the effect of (i) private value, (ii) evolution speed, and (iii) the market fraction of the informed traders 31 We also report the results on the robustness analysis of (iv) the fundamental volatility, (v) the information lag, and (vi) the discount rate of the historical performance in the GA learning in Appendix E.

5.1. Private value. In the BM model, we ignore the trading motivation of the uninformed traders who always lose money to the informed traders 32 which is supported by empirical studies (see Barber, Lee, Liu and Odean (2009) and Chung et al. (2013)). To motivate uninformed traders to trade, Goettler et al. (2009) introduce private value for uninformative traders whose order submissions mainly depend on the private values. For example, an uninformed trader with a large positive private value prefers to submit a market buy order. We are interested in the effect of introducing private values, in particular for the uninformed traders, on the results obtained in the last section.

We consider the uninformed traders who obtain private values (or liquidity compensations) when their orders are executed 33 The private value is considered in the trading performance of the GA according to (11), while the classifier system of their GA learning is kept the same. We consider two cases: a low private value $P V 6$ and

\footnotetext{
${ }^{30} \mathrm{We}$ would like to thank a referee for pointing this connection to momentum trading.

${ }^{31}$ We would like to thank the referees for the suggestion of including the robustness analysis of private value and evolution speed.

${ }^{32}$ Based on the framework of Grossman and Stiglitz (1980), Routledge (1999) allows uninformed traders to make profit; however, the economy considered is quite different from the economy of a dynamic limit order market with continuous double auction.

${ }^{33}$ To keep the model symmetric, independent of buying or selling, the uninformed trader obtains the same private value from his trading.
} 
a high private value $P V 30$. In the case of PV6, the uninformed trader has a private value $p v_{i}$ which is uniformly distributed in $[4,8]$ ticks, which is consistent with Goettler et al. (2009). In the case of PV30 with large private value, $p v_{i}$ is uniformly distributed over $[20,40]$ ticks 34 We keep the private value the same for the trader in each transaction. For example, for an uninformed trader $i$ with a private value of 6 ticks, if the trader submits a market buy order and obtains $p_{t}-v_{t}=-4$, then the order profit of the trader is $6-4=2$ ticks. We find that, compare to the BM, the results are robust when the private value is low (for the case of PV6). However, high private value can have significantly different impact initially, but the impact disappears in long-run. To explore the difference, we report the results based on the first 7,200 periods (10 trading days), denoted by BME and PV30E, and the last 7,200 period after 300 trading days, denoted by BML and PV30L respectively for the BM and PV30 cases. We report the price dynamics of the first 7,200 periods and the last 7,200 periods in Fig. 4. We also report the patterns of information usage of the classifier rules in Fig. 15, the order profit, information efficiency, bid-ask spread in Table 6, and the order submission in Table 7.

TABLE 6. Private value effect on order profit, information efficiency and the bid-ask.

\begin{tabular}{cccccc}
\hline Case & $p v$ & $r_{I}$ & $r_{U}$ & $M R E$ & $s$ \\
\hline BME & 0 & 41.14 & -6.2 & $2.51 \%$ & 5.41 \\
PV30E & {$[20,40]$} & $43.13^{*}$ & $24.84^{*}$ & $2.60 \%^{*}$ & $3.66^{*}$ \\
& & & & & \\
BM & 0 & 35.19 & -3.51 & $2.21 \%$ & 6.24 \\
PV6 & {$[4,8]$} & 35.07 & $2.48^{*}$ & $2.60 \% *$ & $5.13^{*}$ \\
PV30 & {$[20,40]$} & $35.87^{*}$ & $26.38^{*}$ & $2.44 \% *$ & $5.41^{*}$ \\
\hline
\end{tabular}

Figure 4 shows that, compare to the BM case, the private value for the uninformed traders can have a significant impact on the market price initially. This is indicated by large deviations of the market price from the fundamental value for PV30E case most of the time. The corresponding results on order submission in Table 7 show that with private value, the uninformed traders submit more market orders and aggressive limit orders compared to the BM case (comparing BME and PV30E, $M O_{U}$ increases from 109 to 141, and $A L O_{U}$ increases from 60 to 87), and the information efficiency indicated in Table 6 becomes worse than the BM case ( $M R E$ increases from $2.51 \%$ to $2.60 \%$ ), but the spread is reduced (from 5.41 to

\footnotetext{
${ }^{34}$ Please note that in the cases of PV6 and PV30, the private value corresponds to a no-zero-sum game.
} 


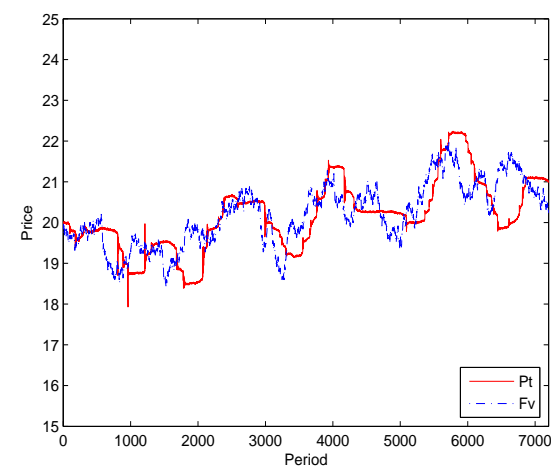

(a) BME

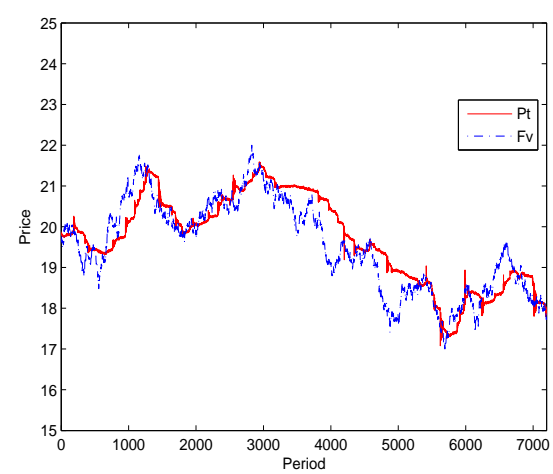

(c)BML

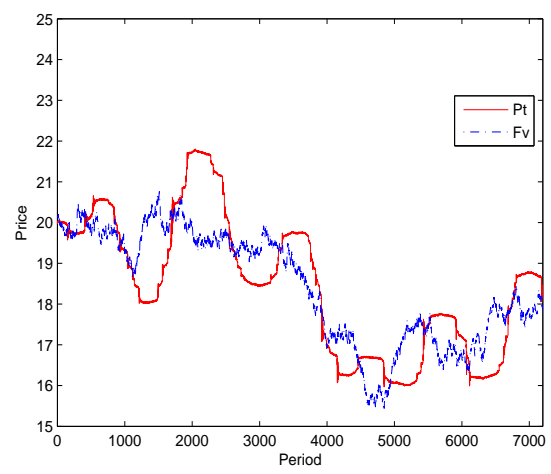

(b) PV30E

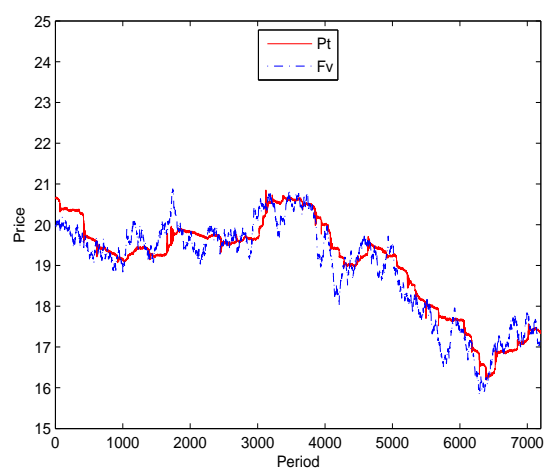

(d)PV30L

FiguRE 4. The market price and fundamental value for the BM case (a) and PV30 case (b) over the first 7,200 periods and the BM case (c) and the PV30 case (d) over the last 7,200 periods.

3.66). Intuitively, since the uninformed traders are compensated from their trading, they trade more aggressively by submitting more market orders, which increase the price volatility and reduce the information efficiency. They also submit more aggressive limit orders, which reduce the spread. However, in long run, the impact of the private values becomes insignificant, compare to the BM case. Fig. 4 shows that the price dynamics of the PV30 case in (d) is similar to the BM case in (c). Fig. 5 shows that the average usages of the five groups of market information under the GA learning for the uninformed traders with low and high private values (in PV6 and PV30 cases) share similar patterns to the BM case in Fig. 3 (b). Table 6 reports that the uninformed traders make positive order profit 35 from their trading in the PV6 and PV30 cases, however, the order profit for the informed traders, together with the market information efficiency and the bid-ask spread, does not change much. In addition, Table 7 reports that the order submissions do not change

\footnotetext{
${ }^{35}$ Taking the mean private values into account, the order profit for the PV6 and PV30 is about the same as the BM case for the uninformed traders
} 


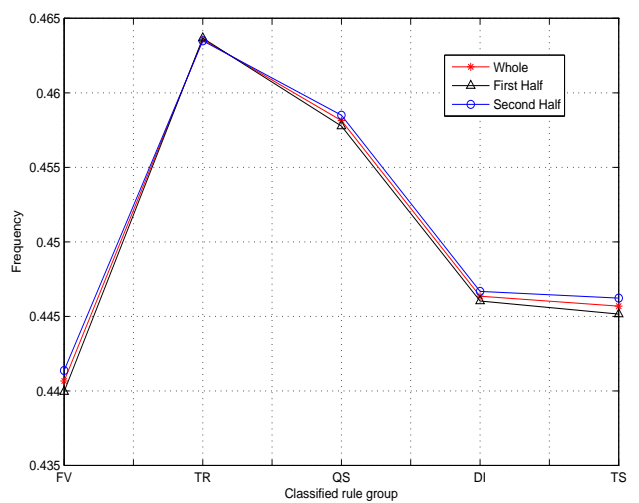

(a) PV6 case

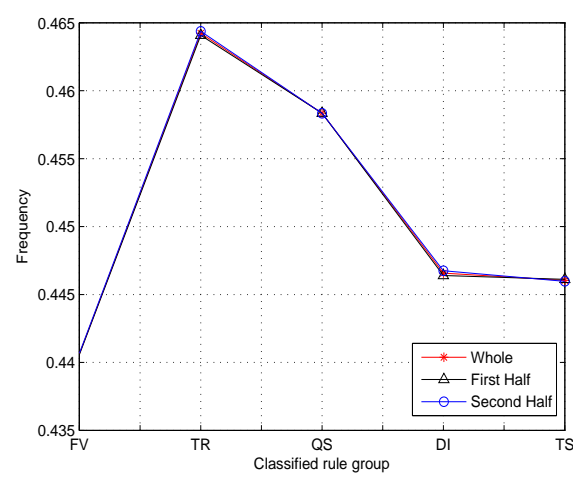

(b) PV30 case

FigurE 5. The patterns of the means of the whole series, the first half and the second half of $\gamma_{i}$ among different classified rule groups of uninformed traders in PV6 case (a) and PV30 case(b).

TABLE 7 . The private value effect on order submission.

\begin{tabular}{c|ccccc}
\hline Case & $M O_{I}$ & $A L O_{I}$ & $L O A_{I}$ & $U L O_{I}$ & $E L O_{I}$ \\
\hline BME & 258 & 29 & 201 & 113 & 70 \\
PV30E & $232^{*}$ & $44^{*}$ & 203 & $120^{*}$ & $95^{*}$ \\
& & & & & \\
BM & 155 & 100 & 172 & 173 & 149 \\
PV6 & 155 & 100 & 172 & 174 & 150 \\
PV30 & 155 & 100 & 172 & 174 & 150 \\
\hline Case & $M O_{U}$ & $A L O_{U}$ & $L O A_{U}$ & $U L O_{U}$ & $E L O_{U}$ \\
\hline BME & 109 & 60 & 225 & 206 & 130 \\
PV30E & $141^{*}$ & $87^{*}$ & $198^{*}$ & $174^{*}$ & $157^{*}$ \\
& & & & & \\
BM & 169 & 96 & 167 & 168 & 170 \\
PV6 & 169 & 94 & 168 & 169 & 170 \\
PV30 & 169 & 94 & 168 & 169 & 169 \\
\hline
\end{tabular}

significantly. Therefore the private value of the uninformed traders may have significant impact on the limit order market and order submission in short run, but the impact becomes insignificant in long run. This is very different from Goettler et al. (2009) in which the order submission of uninformed traders depends on their private values. With the GA learning, the order submission and order profit (after taking their compensation into account) of the uninformed traders do not depend on their private values. 
5.2. The evolution speed of the GA. It is important to understand how the speed of the evolution of the GA learning affects the results. In some GA learning models, such as SFI-ASM, the evolution speed has impact on the convergence of GA and market dynamics. In the BM case, the evolution of GA takes asynchronously on average of 360 periods (one trading day). To examine this effect, we consider two cases to examine the effect of slow down in the evolution of GA 36 In the first case, we reduce the evolution speed to 720 periods (two trading days) on average, denoted by SD2, which is two times slower than the BM case; in the second case, we reduce the evolution speed to 1,800 periods ( 5 trading days) on average, denoted by SD5, which is 5 times slower than the BM case. For the SD2 case, Fig. 6 (a) and (b) show that the evolution dynamics of $\gamma_{i}$ do not change significantly. The changes in order profit, information efficiency and the bid-ask spread are also small, showing in Table 8. Intuitively, when the evolution speed of the GA reduces (but not too much), trading strategies have more opportunity to be selected and tested and therefore better performed strategies are more likely to be selected for the evolution in the next generation. However, if the evolution speed slows down substantially, such as in the SD5 case, the GA may not be able to converge (even after 500 trading days), illustrated in Fig. 6(c) and (d), and the market dynamics and the order submission reported in Tables 8 and 9 change significantly. Therefore there is a trade-off effect between the speed of the evolution and the effectiveness of the GA learning.

TABLE 8. Slow evolution effect on order profit, information efficiency and the bid-ask spread.

\begin{tabular}{ccccc}
\hline Case & $r_{I}$ & $r_{U}$ & $M R E$ & $s$ \\
\hline BM & 35.19 & -3.51 & $2.21 \%$ & 6.24 \\
SD2 & $36.80^{*}$ & $-3.67^{*}$ & $2.34 \%^{*}$ & $5.84^{*}$ \\
SD5 & $19.07^{*}$ & $-4.33^{*}$ & $0.79 \%$ & $5.36^{*}$ \\
\hline
\end{tabular}

\subsection{The fraction of informed traders and the implication for PIN. Clearly} the market fraction of the informed traders, the fundamental volatility, the information lag and the weight on the last performance can affect the results. We conduct a robustness analysis of these factors and report the results in Appendix E. The results show in all the cases, the GA learning process becomes stationary in the long run, the patterns of usage frequency among classified rule groups are similar, and the effects on the order profit, information efficiency and the order submission are consistent with our intuition (for example, the information efficiency, the spread

\footnotetext{
${ }^{36}$ This robustness test is suggested by an anonymous referee.
} 


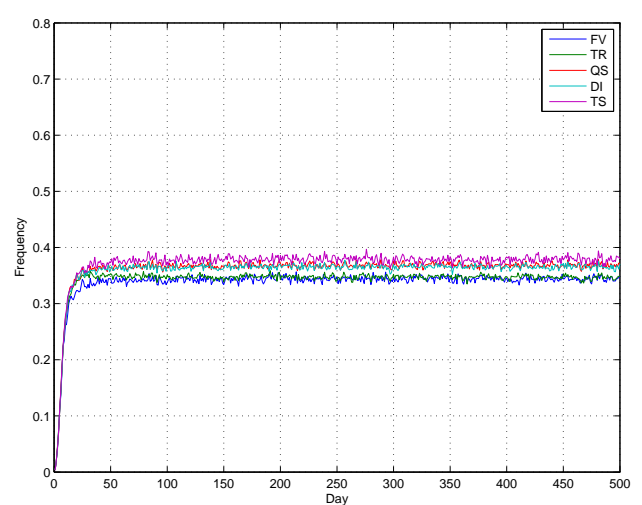

(a) Informed traders in SD2

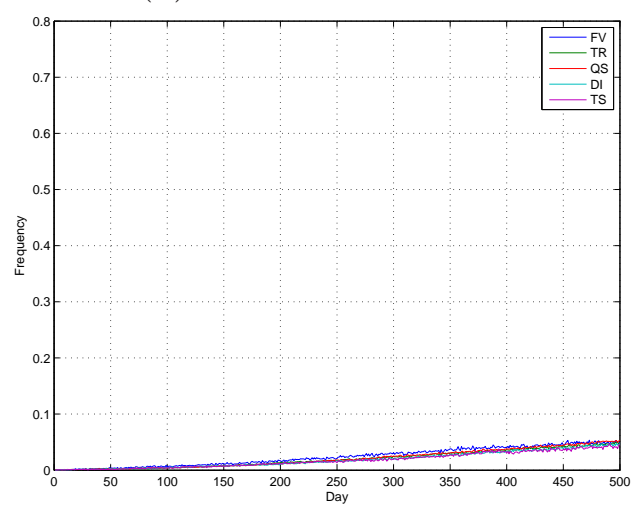

(a) Informed traders in SD5

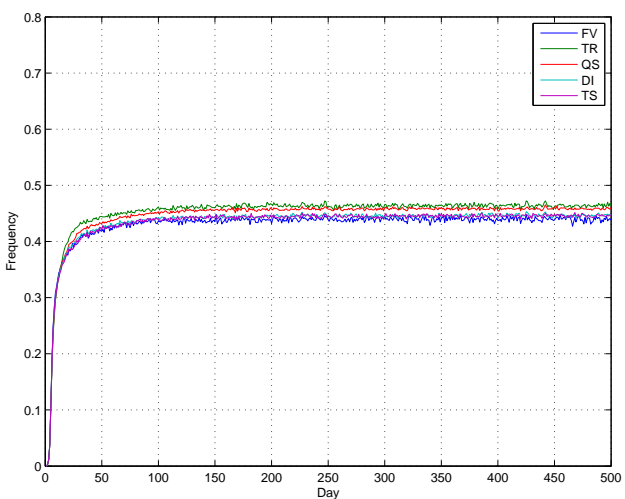

(b) Uninformed traders in SD2

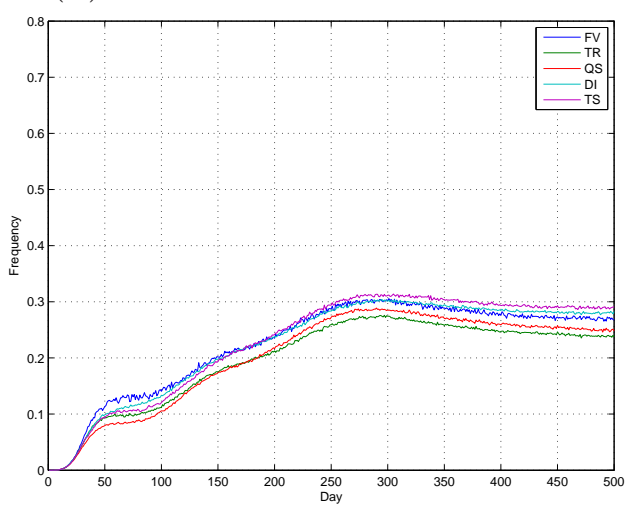

(b) Uninformed traders in SD5

Figure 6. The evolution dynamics of $\gamma_{i}$ in the SD2 case and the SD5 case.

TABLE 9. Slow evolution effect on order submission.

\begin{tabular}{c|ccccc}
\hline Case & $M O_{I}$ & $A L O_{I}$ & $L O A_{I}$ & $U L O_{I}$ & $E L O_{I}$ \\
\hline BM & 155 & 100 & 172 & 173 & 149 \\
SD2 & 155 & 100 & 171 & 173 & 149 \\
SD3 & $485^{*}$ & $36^{*}$ & $59^{*}$ & $19^{*}$ & $28^{*}$ \\
\hline Case & $M O_{U}$ & $A L O_{U}$ & $L O A_{U}$ & $U L O_{U}$ & $E L O_{U}$ \\
\hline BM & 169 & 96 & 167 & 168 & 170 \\
SD2 & 169 & 95 & 168 & 169 & 170 \\
SD5 & $75^{*}$ & $70^{*}$ & $305^{*}$ & $150^{*}$ & $126^{*}$ \\
\hline
\end{tabular}

and the order profit of the informed traders improve as the fraction of the informed traders decreases).

The robustness analysis on the fraction of the informed traders has an implication to the probability of informed trading (PIN), which is a very important measure in limit order markets. Both the spread and volatility are very important indicators for the PIN estimation. In our model, we can calculate the PIN directly since we know 
the executed orders of informed traders exactly. As the fraction of the informed traders increases from $5 \%$ to $90 \%$, we report the PIN, volatility of market-price return (in basis points), bid-ask spread (in ticks), and trading volume in Table 10. The results show that the calculated PIN is matching closely to the fraction of the informed traders. Also, when the fraction of the informed traders increases, both the spread and volatility increase, while the trading volume decreases, and the relation are monotonic but nonlinear 37 The positive relation between the PIN and the spread is consistent with some empirical studies in the environment of short-lived information (see Ahn, Cai, Hamao and Ho (2002) and Ruchti (2012)), but different from Rosu (2014) who infers that the fraction of informed traders and the PIN are negatively correlated to the bid-ask spread when the information is long-lived. Rosu (2014) also suggests to use the ratio of the market-price volatility to the bid-ask spread to measure the informed shares, which can be represented by the PIN. We also report the ratio of the volatility to the bid-ask spread in Table10. Different from Rosu (2014), the result shows that the PIN is positively correlated with the bid-ask spread and the volatility, but negatively correlated with the ratio of the volatility to the bid-ask spread. Therefore it is very important to consider the environment of short/long-lived information when using various measures to estimate the PIN 38 Since the volatility of market-price return and the bid-ask spread are observable from the intra-day data, this result provides a useful implication for the estimation of the PIN, in particular for intra-day PIN models in limit order markets 39

To conclude the discussion, we provide some intuitions on the above findings. As the number of the informed traders increases, on the one hand, any mispriced limited order from the uninformed traders is more likely executed by market orders from the informed traders, which enlarges the spread and increases the volatility. On the other hand, the pick-off risk increases for the informed traders due to the competition among themselves; hence they prefer to use market orders or less aggressive limit orders instead of aggressive limit orders when trading is profitable. This leads to an

\footnotetext{
${ }^{37}$ We thank an anonymous referee for the suggestion of examining the monotonicity.

${ }^{38}$ Rosu (2014) also pointed out that: "It is possible that both views are correct, but in different circumstances. For instance, one can expect the adverse selection view to hold in the case of scheduled corporate announcements, when information is relatively short-lived. At the same time, the dynamic efficiency view may hold in a more stationary environment, with non-scheduled news and long-lived information. Each view is supported by empirical work."

${ }^{39}$ The extant PIN models are based on three types of model including Easley, Keifer, OHara and Paperman (1996) who estimate the PIN of daily frequency based on aggregate buy-sell initiated trades in market maker markets; Nyholm (2003) who estimates the PIN with high frequency based on the bid-ask spread set by market makers; and Handa, Schwartz and Tiwari (2003) who estimate the PIN based on the bid-ask spread in limit order markets (by assuming that informed traders only submit market orders).
} 
increase in the spread and volatility. In addition, when more informed traders arrive the market during a trading period, their orders are more likely on the same side of the book, which reduce the trading volume and increase the spread. However, when the bid-ask spread is too large, the learning makes traders submit more aggressive limit orders (see the ALO in Table 15). This effect narrows the bid-ask spread. Therefore the monotonic relations between the bid-ask spread, volatility, volume and the fraction of the informed traders are nonlinear.

TABLE 10. The volatility of market-price return in basis points, the bid-ask spread in ticks and the probability of informed trading.

\begin{tabular}{cccccc}
\hline Fraction & PIN & Volatility & Spread & Volatility/Spread & volume \\
\hline $5 \%$ & $4.70 \% *$ & $28.70^{*}$ & $4.38^{*}$ & $6.55^{*}$ & 4.7 \\
$10 \%$ & $9.06 \%$ & 39.28 & 6.24 & 6.29 & 4.7 \\
$20 \%$ & $17.53 \%^{*}$ & $49.19^{*}$ & $8.53^{*}$ & $5.77^{*}$ & $4.6^{*}$ \\
$50 \%$ & $43.56 \%^{*}$ & $56.88^{*}$ & $10.83^{*}$ & $5.25^{*}$ & $4.4^{*}$ \\
$90 \%$ & $86.98 \%^{*}$ & $69.31^{*}$ & $13.41^{*}$ & $5.17^{*}$ & $4.1^{*}$ \\
\hline
\end{tabular}

\section{Conclusion}

What traders can learn from the market and how they process market information are very important and challenging questions in limit order markets. This paper proposes a dynamic limit order model in which the trading strategies of the informed and uninformed traders evolve endogenously. By introducing a genetic algorithm learning with a classifier system, we combine information processing and order choice into the trading strategy and allow traders to endogenously determine their order submission according to market conditions. This helps to overcome the challenge and reveal the black box of information processing and to examine the learning effect on market efficiency and order submission .

We show that when the evolution of trading strategies becomes stationary in long run, the average information usage is higher for uninformed traders than informed traders. Also informed traders pay more attention to the last transaction sign while uninformed traders pay more attention to technical rules (in particular the moving average rule of historical prices) than other market information. Learning of uninformed traders reduces their loss, improves market information efficiency and reduces the bid-ask spread while learning of informed traders improves their order profit, reduces bid-ask spread but the market information efficiency may be reduced. In general, learning is more effective and hence more valuable to uninformed traders than informed traders. Learning makes uninformed traders submit more market 
orders than aggressive limit orders while learning makes informed traders reduce market orders and increase aggressive limit orders.

We also conduct a robustness analysis and provide some implications. Surprisedly, we find that though private value can influence the order submission of uninformed traders at early periods, however, in the long run, its impact is ultimately eliminated by the GA learning so it does not have impact on the learning efficiency and order submission. Interestingly, we find that the evolution speed does not have significant impact on the learning and order submission when it does not slow down too much. More importantly, we find that the probability of informed trading (PIN) is positively correlated with volatility and the bid-ask spread but negatively correlated with the ratio of volatility to the bid-ask spread. This is a useful implication for estimating the PIN in limit order markets.

Our model can be expanded in at least two aspects in the future research. One aspect is that improving the classifier system and let traders learn from more order book information. The other aspect is that using GA learning to capture the features of high frequency trading in limit order markets. 


\section{APPENDIX}

A. Some special limit order book scenarios. Obviously, in some special limit order book scenarios, some actions are impossible or unused. For example, when the bid-ask spread is minimum (equal to one tick), a trader can not submit aggressive limit orders; and when the buy (sell) side limit order book is empty, a trader can not submit market sell (buy), and if he/she submits a limit buy (sell), the submitted limit order becomes the limit order at quote at the buy (sell) side. We list the scenarios and corresponding actions in Table 11 .

TABLE 11. The restrictions of actions.

\begin{tabular}{c|c|c}
\hline \multicolumn{2}{|c}{ Scenario } & Unused action \\
\hline The book is not empty & $\begin{array}{c}\text { The bid-ask spread is more than one tick } \\
\text { The bid-ask spread is equal to one tick }\end{array}$ & None \\
\hline The book is empty & Only when the buy side is empty & MS, ALB \& ULB \\
& Only when the sell side is empty & MB, ALS \& ULS \\
& Both the buy and sell sides are empty & MB, ALB, ULB, MS, ALS \& ULS \\
\hline
\end{tabular}

B. The stationarity of trading strategies. In this appendix, we provide statistical analysis to examine the stationarity of trading strategies, which is measured by the average usage frequency of different group of market information. To examine whether the mean of $\gamma_{i}$ is stationary, we select the $\gamma_{i}$ of the last 200 generations (from the generation 301 to the generation 500) and split the whole series into two parts with 100 equal generations. At first step, we estimate the distribution of $\gamma_{i}$. Fig. 7 reports the distribution plots for $\gamma_{T S}$ of informed traders in (a) and $\gamma_{T R}$ of uninformed traders in (b) respectively. Fig. 7 shows that the distribution of $\gamma_{i}$ approximates a normal distribution. 40

We also use ANOVA (Analysis of Variance) to examine whether the first half has the same mean as the second half. We report the probability of ANOVA in Table 12 (see the last row). We also report the mean, maximum, minimum and standard deviation of the whole series. It shows that the probability of ANOVA of each $\gamma_{i}$ for both informed and uninformed traders is higher than $1 \%$ (for most $i, \gamma_{i}$ is higher than 5\%). Therefore the ANOVA can not reject the hypothesis and demonstrates that the first half and the second half series have the same mean. Also the maximum and minimum of $\gamma_{i}$ are close and the standard deviation is very small. The result shows that $\gamma_{i}$ becomes a stationary in long run.

\footnotetext{
${ }^{40} \mathrm{We}$ also estimate the distributions of the other $\gamma_{i}$ and the results are the same.
} 


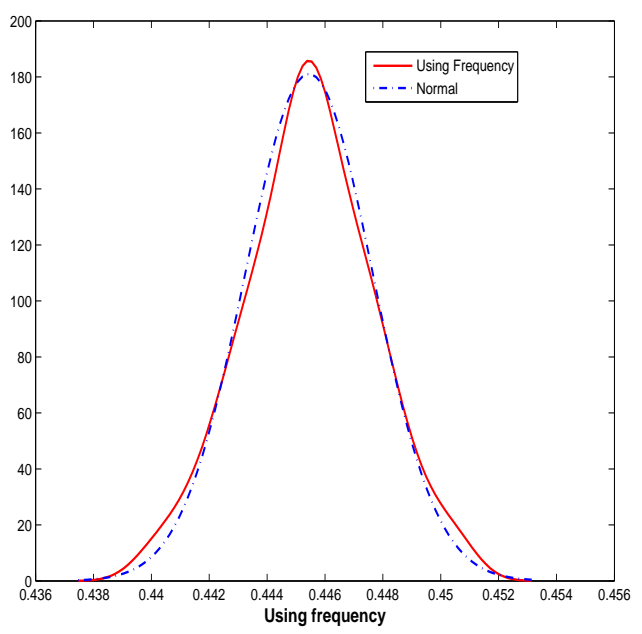

(a) Informed traders

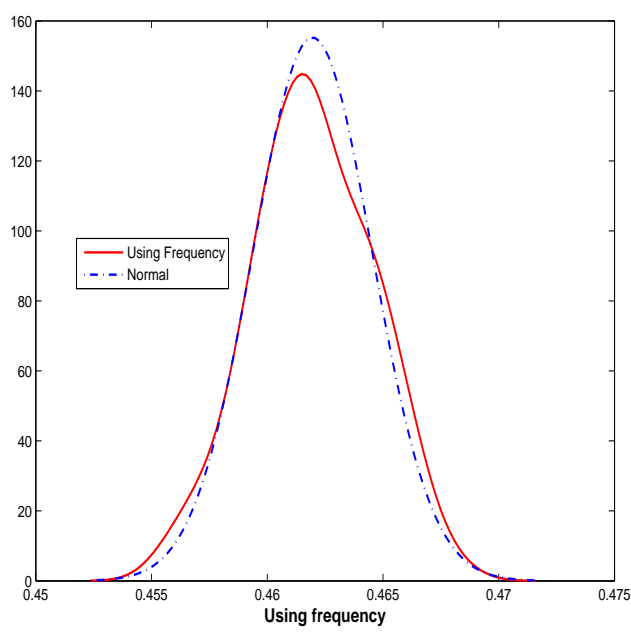

(b) Uninformed traders

FiguRE 7. The estimated distribution plots of (a) $\gamma_{T S}$ of informed traders and (b) $\gamma_{T R}$ of uninformed traders.

TABLE 12. The statistics of $\gamma_{i}$ in the last 200 generations (200 trading days, 72,000 periods). The last column is the probability of ANOVA for the first half and the second half of the series.

\begin{tabular}{cccccc}
\hline Group & Mean & MAX & MIN & STD & ANOVA \\
\hline Informed & traders & & & & \\
FV & 0.343 & 0.354 & 0.330 & 0.005 & $97.33 \%$ \\
TR & 0.351 & 0.360 & 0.339 & 0.004 & $22.25 \%$ \\
QS & 0.365 & 0.374 & 0.356 & 0.004 & $51.26 \%$ \\
DI & 0.362 & 0.373 & 0.353 & 0.004 & $21.73 \%$ \\
TS & 0.375 & 0.390 & 0.363 & 0.005 & $73.17 \%$ \\
\hline Uninfromed & traders & & & & \\
FV & 0.440 & 0.452 & 0.429 & 0.003 & $90.57 \%$ \\
TR & 0.462 & 0.468 & 0.456 & 0.003 & $9.75 \%$ \\
QS & 0.457 & 0.462 & 0.452 & 0.002 & $4.22 \%$ \\
DI & 0.446 & 0.451 & 0.440 & 0.002 & $11.95 \%$ \\
TS & 0.445 & 0.451 & 0.440 & 0.002 & $45.66 \%$ \\
\hline
\end{tabular}

C. The ANOVA for the patterns of usage frequency among CR groups. We provide the ANOVA of the patterns of $\gamma_{i}$ among different CR groups for informed traders in Figure 8(a) and for uninformed traders in Figure 8(b). We also report the the ANOVA of the patterns of among different Classified rules (from CR1 to CR10) for uninformed traders in Figure 9 . 


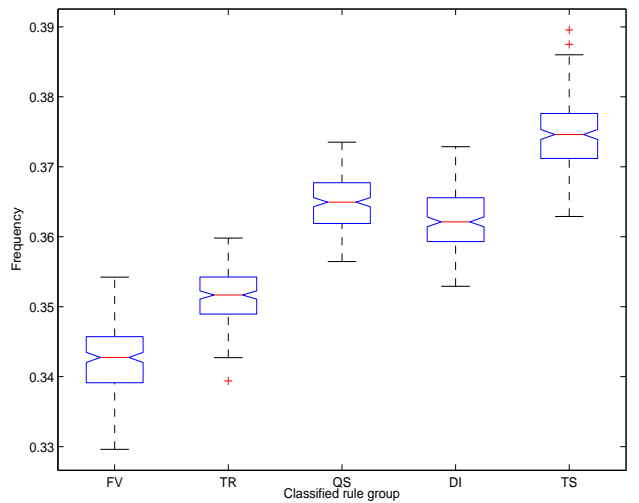

(a) Informed traders

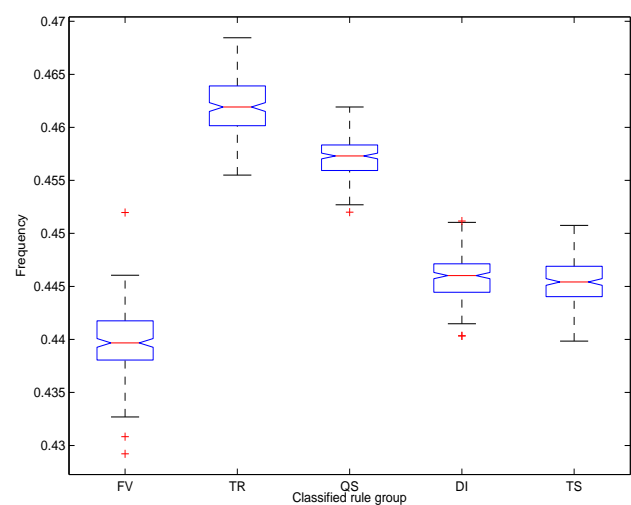

(b) Uninformed traders

Figure 8. The ANOVA of $\gamma_{i}$ among the CR groups of the last 100 generations for (a) informed and (b) traders. The $p$ value is 0 .

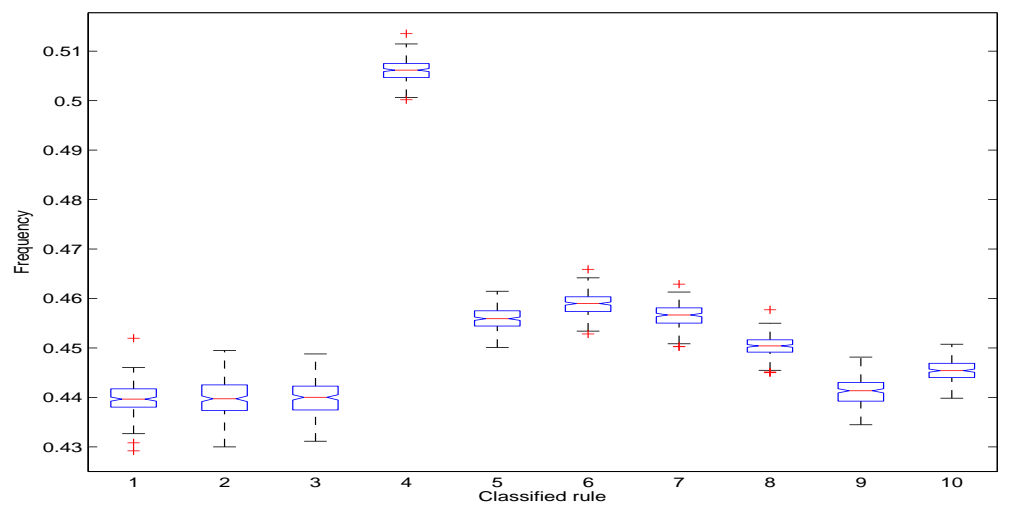

Figure 9. The ANOVA of average usage frequency among different CRs of the last 200 generations for uninformed traders. The $p$ value is 0 .

D. The effect of classified information on order submission. We report the order submission for the Group TS of informed trades and the Group TR of uninformed traders in Table 13 .

E. Other robustness tests. Based on Section 5, we conduct a further robustness analysis including (i) cases $L I$ and $H I$ for lower and higher number of informed trades with $N_{I}=50$ and $N_{I}=200$ respectively (meaning the market fraction of the informed traders is $5 \%$ for $L I$ case, $10 \%$ for the BM case, and $20 \%$ for $H I$ case); (ii) case $L V$ for lower fundamental volatility $\kappa=2$ (meaning that the fundamental value changes two ticks, instead of four ticks for the BM case, per minute on average); (iii) case $S L$ for short information lag $\tau=180$ (half day, instead of one day in the $\mathrm{BM}$ case); and (iv) case $L B$ for lower weight $\beta=0.05$ (instead of $\beta=0.2$ for the BM case) on the recent profit in the performance measure of the GA. The 
TABLE 13. The order submission for the Group TS of informed trades and the Group TR of uninformed traders.

\begin{tabular}{cccccc}
\hline Case & $M O_{I}$ & $A L O_{I}$ & $L O A_{I}$ & $U L O_{I}$ & $E L O_{I}$ \\
\hline BM & 155 & 100 & 172 & 173 & 149 \\
& {$[1]$} & {$[2]$} & {$[2]$} & {$[2]$} & {$[2]$} \\
NTS & $151^{*}$ & 101 & 173 & 175 & 149 \\
& {$[2]$} & {$[3]$} & {$[2]$} & {$[2]$} & {$[2]$} \\
NTR & 155 & 100 & 172 & 173 & 151 \\
& {$[2]$} & {$[3]$} & {$[2]$} & {$[2]$} & {$[2]$} \\
\hline Case & $M O_{U}$ & $A L O_{U}$ & $L O A_{U}$ & $U L O_{U}$ & $E L O_{U}$ \\
\hline BM & 169 & 96 & 167 & 168 & 170 \\
& {$[1]$} & {$[3]$} & {$[1]$} & {$[1]$} & {$[1]$} \\
NTS & 169 & 96 & 167 & 168 & 169 \\
& {$[1]$} & {$[3]$} & {$[1]$} & {$[1]$} & {$[1]$} \\
NTR & 169 & $93^{*}$ & 169 & 169 & 170 \\
& {$[1]$} & {$[3]$} & {$[1]$} & {$[1]$} & {$[1]$} \\
\hline
\end{tabular}

other parameters are the same as the BM case. We first examine the stationarity of trading strategies and then the effect of learning and information.

The stationarity of trading strategies-For all these cases, we find the same stationarity of trading strategies as in the BM case. We report the patterns of the average usage frequencies among different classified rule groups for informed traders and uninformed traders in Fig. 10 (a) and (b) respectively. We see that Fig. 10 shares the same patterns as in Fig. 3, that is, the information usage is higher for the uninformed traders than the informed traders, also the most used information classified rule is the last transaction sign (TS) for the informed traders and the technical rules (TR) for the uninformed traders. Comparing among different cases, we find from Fig.10 (a) that, for the informed traders, the average usage of the CR groups QS, DI and TS are the highest for case $L I$, while the lowest is group TR for case $S L$. This indicates that, when there are less informed traders, the market becomes less informative and the informed traders pay more attention to the order book information in order to make profit from trading with uninformed traders. When the information lag is short, informed traders pay less attention to technical rules. From Fig:10 (b), we find that for the uninformed traders, the average usage of all the classified rule groups is the highest for case $S L$. This means that, when the information lag is short, market becomes more informative and the uninformed traders use all the market information more frequently in order to reduce their loss. 


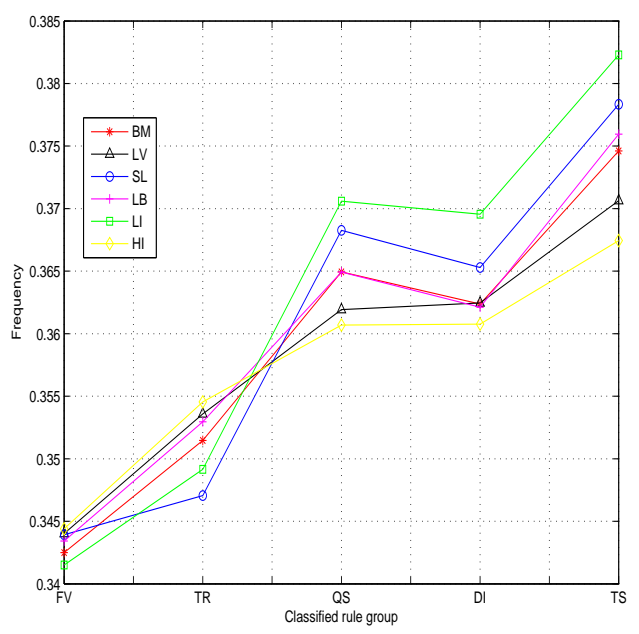

(a) Informed traders

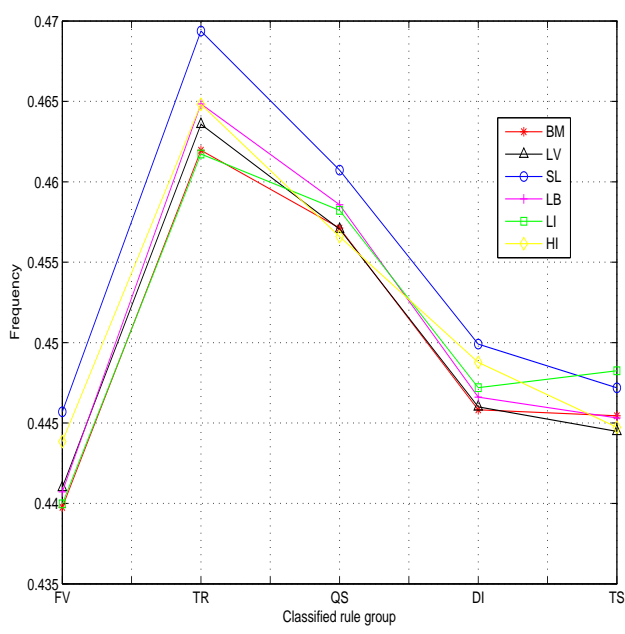

(b) Uninformed traders

Figure 10. The patterns of the means of different cases of $\gamma_{i}$ among different classified rule groups for (a) informed and (b) uninformed traders.

The effect on order profit and information efficiency-We report the results of the order profit, market information efficiency, and bid-ask spread for all the five cases in Table 14. Comparing to the BM model, it shows a significant impact of the volatility (case $L V$ ) and the number of the informed traders (cases $L I$ and $H I$ ) but insignificant impact of the information lag (case $S L$ ) and the performance measure (case $L B$ ). In particular, when the volatility is reduced (from $\kappa=4$ in the $B M$ case to $\kappa=2$ in case $L V$ ), market becomes more informative, which reduces the average order profit by about $60 \%$ (from 35.19 to 12.83 ticks) for the informed traders and the loss by about $60 \%$ for the uninformed traders. In addition, the market efficiency improves significantly and the spread becomes smaller. When the number of the informed traders drops (from $10 \%$ in the BM to $5 \%$ in case $L I$ ), market becomes less informative. The profit for the informed traders increases by about $63 \%$ (because of their information advantage) and the loss for the uninformed traders is also reduced by about $6 \%$ (due to the learning). Both the market efficiency and spread are reduced. When the number of informed traders increases (from 10\% in the BM to $20 \%$ in case $H I$ ), the effect becomes opposite (except that the loss for the uninformed traders is reduced) but is less significant.

The effect on order submission - We report the results of order submission of the informed and uninformed traders for all the five cases in Table 15. Similar to the previous discussion, the fundamental volatility (case $L V$ ) and the number of informed traders (cases $L I$ and $H I$ ) have some significant impacts on the order submission for both the informed and uninformed traders; however, the information 
TABLE 14. The effect on order profit, information efficiency and bidask spread

\begin{tabular}{ccccc}
\hline Case & $r_{I}$ & $r_{U}$ & $M R E$ & $s$ \\
\hline BM & 35.19 & -3.51 & $2.21 \%$ & 6.24 \\
& {$[4.61]$} & {$[0.45]$} & {$[1.37 \%]$} & {$[2.91]$} \\
LV & $12.83^{*}$ & $-1.30^{*}$ & $0.77 \% *$ & $4.77^{*}$ \\
& {$[1.17]$} & {$[0.12]$} & {$[0.36 \%]$} & {$[1.38]$} \\
SL & 34.89 & -3.50 & $2.42 \%$ & 5.64 \\
& {$[3.92]$} & {$[0.39]$} & {$[1.54 \%]$} & {$[2.63]$} \\
LB & 35.56 & -3.55 & $2.56 \%$ & 5.96 \\
& {$[4.58]$} & {$[0.45]$} & {$[1.60 \%]$} & {$[3.54]$} \\
LI & $67.27^{*}$ & -3.31 & $3.90 \% *$ & $4.38^{*}$ \\
& {$[15.87]$} & {$[0.77]$} & {$[2.42 \%]$} & {$[1.26]$} \\
HI & $15.07^{*}$ & $-3.19^{*}$ & $1.00 \% *$ & $8.53^{*}$ \\
& {$[0.64]$} & {$[0.13]$} & {$[0.57 \%]$} & {$[3.42]$} \\
\hline
\end{tabular}

lag (case $S L$ ) only affects the order submission of uninformed (not informed) traders significantly.

As the volatility increases (from $\kappa=2$ in case $L V$ to $\kappa=4$ in the BM case), the informed traders submit less market orders $\left(M O_{I}\right)$ and more aggressive limit orders $\left(A L O_{I}\right)$, while the uninformed traders also submit more aggressive limit orders $\left(A L O_{U}\right)$ but less unaggressive limit orders $\left(U L O_{U}\right)$. Note that the executed limit orders decrease significantly for the informed traders (see $E L O_{I}$ ), but not for the uninformed traders (see $E L O_{U}$ ); also more limit orders from the uninformed traders, instead of the informed traders, are executed. The result is different from Goettler et al. (2009) who find that when volatility is higher, informed traders submit more market orders but less limit orders.

As the number of the informed traders increases (from $5 \%$ in case $L I$ to $10 \%$ in the $\mathrm{BM}$ and then to $20 \%$ in case $H I$ ), both the informed and uninformed traders submit more aggressive limit orders $(A L O)$ but less unaggressive limit orders $(U L O)$ and the limit orders at quote $(L O A)$; also only the uninformed (not informed) traders submit less market orders (see $M O_{U}$ ). Note that the executed limit orders are reduced for the informed traders significantly but increased for the uninformed traders with more limit orders from the uninformed traders are executed. One implication of this result is that, when the number of informed traders increases, there are more aggressive limit orders and less market orders and limit orders at or away the quote. 
TABLE 15. The effect on order submission.

\begin{tabular}{c|ccccc}
\hline Case & $M O_{I}$ & $A L O_{I}$ & $L O A_{I}$ & $U L O_{I}$ & $E L O_{I}$ \\
\hline BM & 155 & 100 & 172 & 173 & 149 \\
& {$[1]$} & {$[2]$} & {$[2]$} & {$[2]$} & {$[2]$} \\
LV & $158^{*}$ & $97^{*}$ & 172 & 174 & $152^{*}$ \\
& {$[2]$} & {$[2]$} & {$[2]$} & {$[1]$} & {$[2]$} \\
SL & 156 & 99 & 172 & 174 & 148 \\
& {$[1]$} & {$[3]$} & {$[2]$} & {$[2]$} & {$[2]$} \\
LB & 155 & 100 & 171 & 174 & 149 \\
& {$[1]$} & {$[3]$} & {$[2]$} & {$[2]$} & {$[2]$} \\
LI & 156 & $98^{*}$ & 173 & 174 & $163^{*}$ \\
& {$[2]$} & {$[2]$} & {$[2]$} & {$[2]$} & {$[2]$} \\
HI & 154 & $106^{*}$ & $169^{*}$ & $171^{*}$ & $132^{*}$ \\
& {$[1]$} & {$[3]$} & {$[2]$} & {$[1]$} & {$[2]$} \\
\hline Case & $M O_{U}$ & $A L O_{U}$ & $L O A_{U}$ & $U L O_{U}$ & $E L O_{U}$ \\
\hline BM & 169 & 96 & 167 & 168 & 170 \\
& {$[1]$} & {$[3]$} & {$[1]$} & {$[1]$} & {$[1]$} \\
LV & 170 & $93^{*}$ & 168 & $170^{*}$ & 170 \\
& {$[1]$} & {$[2]$} & {$[1]$} & {$[1]$} & {$[1]$} \\
SL & $165^{*}$ & 96 & $171^{*}$ & $169^{*}$ & $165^{*}$ \\
& {$[2]$} & {$[3]$} & {$[2]$} & {$[1]$} & {$[1]$} \\
LB & 169 & 95 & 168 & 169 & 170 \\
& {$[1]$} & {$[3]$} & {$[1]$} & {$[1]$} & {$[1]$} \\
& $171^{*}$ & $92^{*}$ & $168^{*}$ & $170^{*}$ & 170 \\
& {$[1]$} & {$[2]$} & {$[1]$} & {$[1]$} & {$[1]$} \\
& $166^{*}$ & $102^{*}$ & $165^{*}$ & $166^{*}$ & $172^{*}$ \\
& {$[1]$} & {$[3]$} & {$[2]$} & {$[1]$} & {$[1]$} \\
\hline
\end{tabular}

Comparing the BM case to case $S L$, we find that, when the information lag increases, the uninformed traders submit more market orders $\left(M O_{U}\right)$ and less unaggressive limit orders $\left(U L O_{U}\right)$ and limit orders at quote $\left(L O A_{U}\right)$. Also, more limited orders from the uninformed traders are executed (see $E L O_{U}$ ). This implies that, when the information lag becomes longer, liquidity consumption increases, liquidity supply decreases and this change in liquidity is mainly from uninformed traders. Finally, we find that the weight of the recent profit does not have significant impact. 


\section{REFERENCES}

Ahn, H.-J., Cai, J., Hamao, Y. and Ho, R. Y. K. (2002), 'The components of the bid-ask spread in a limit order market: evidence from the tokyo stock exchange', Journal of Empirical Finance 9, 399-430.

Allen, T. W. and Carroll, C. D. (2001), 'Individual learning about consumption', Macroeconomic Dynamics 5(2), 255-271.

Anufriev, M., Arifovich, J., Ledyard, J. and Panchenko, V. (2013), 'Efficiency of continuous double auctions under individual evolutionary learning with full or limited information', Journal of Evolutionary Economics 23, 539-573.

Arifovic, J. (1996), 'The behavior of the exchange rate in the genetic algorithm and experimental economies', Journal of Political Economy 104, 510-541.

Arthur, W., Holland, H., LeBaron, B., Palmer, R. and Tayler, P. (1997), ' Asset pricing under endogenous expectations in an artificial stock market', in The Economy As An Evolving Complex System II, Eds. W. Arthur and S. Durlauf and D. Lane, Addison-Wesley, pp. 15-44.

Barber, B. M., Lee, Y.-T., Liu, Y.-J. and Odean, T. (2009), 'Just how much do individual investors lose by trading?', Review of Financial Studies 22, 609-632.

Bloomfield, R., O'Hara, M. and Saar, G. (2005), 'The 'make or take' decision in an electronic market: evidence on the evolution of liquidity', Journal of Financial Economics 75(1), 165199.

Brock, W. and Hommes, C. (1998), 'Heterogeneous beliefs and routes to chaos in a simple asset pricing model', Journal of Economic Dynamics and Control 22, 1235-1274.

Chakrabarti, R. and Roll, R. (2012), 'Learning from others, reacting and market quality', Journal of Financial Markets 2, 153-178.

Chakravarty, S. and Holden, C. (1995), 'An integrated model of market and limit orders', Journal of Financial Intermediation 4, 213-241.

Chen, S.-H., Chang, C.-L. and Du, Y.-R. (2012), 'Agent-based economic models and econometrics', Knowledge Engineering Review 27(2), 187-219.

Chiarella, C., He, X., Shi, L. and Wei, L. (2014), A behavioural model of investor sentiment in limit order markets, Working Paper 342, Quantitative Finance Research Centre (QFRC), University of Technolgy Sydney.

Chiarella, C., Iori, G. and Perellò, J. (2009), 'The impact of heterogeneous trading rules on the limit order book and order flows', Journal of Economic Dynamics and Control 33(3), 525-537.

Chung, H., Gao, C., Lu, J. and Mizrach, B. (2013), 'An empirical analysis of the shanghai and shenzhen limit order books', Economic Modeling 34, 37-41.

Collin-Dufresne, P. and Fos, V. (2013), Do prices reveal the presence of informed trading?, Working paper, SSRN.

Comerton-Forde, C. and Putniṇš, T. J. (2013), Dark trading and price discovery, Working paper, SSRN.

Darley, V. and Outkin, A. V. (2007), A NASDAQ Market Simulation, Complex Systems and Interdisciplinary Science Volume 1, World Scientific.

Dugast, J. (2012), Limit attention and news arrival in limit order markets, Working paper, HEC Paris. 
Easley, D., Keifer, N., O’Hara, M. and Paperman, J. (1996), 'Liquidity, information, and infrequently traded stocks', Journal of Finance 51, 1405-1436.

Glosten, L. R. (1994), 'Is the electronic open limit order book inevitable?', Journal of Finance 49(4), 1127-1161.

Goettler, R. L., Parlour, C. A. and Rajan, U. (2005), 'Equilibrium in a dynamic limit order market', Journal of Finance 10, 2149-2192.

Goettler, R. L., Parlour, C. A. and Rajan, U. (2009), 'Informed traders and limit order markets', Journal of Financial Economics 93(1), 67-87.

Gould, M., Porter, M., Williams, S., Fenn, D. and Howison, S. D. (2013), 'Limit order books', Quantitative Finance 13(11), 1709-1742.

Grinblatt, M., Keloharju, M. and Linnainma, J. T. (2012), 'IQ, tradingbehavior,and performance', Journal of Financial Economics 104, 339-362.

Grossman, S. and Stiglitz, J. (1980), 'On the impossibility of informationally efficient markets', American Economic Review 70, 393-408.

Handa, P., Schwartz, R. and Tiwari, A. (2003), 'Quote setting and price formation in an order driven market', Journal of Financial Markets 6(4), 461-489.

Harris, L. (1998), 'Optimal dynamic order submission strategies in some stylized trading problems', Financial Markets, Institutions, and Instruments 7, 1-76.

Holland, J. H. (1975), Adaptation in Natural and Artificial Systems: An Introductory Analysis with Applications to Biology, Control, and Artificial Intelligence, University of Michigan Press, Ann Arbor, MI.

Kaniel, R. and Liu, H. (2006), 'What orders do informed traders used?', Journal of Business 79, 1867-1913.

Keim, D. and Madhavan, A. (1995), 'Anatomy of the trading process: Empirical evidence on the behavior of institutional traders', Journal of Financial Economics 37, 371-398.

Kluger, B. D. and McBride, M. E. (2011), 'Intraday trading patterns in an intelligent autonomous agent-based stock market', Journal of Economic Behavior \& Organization 79(3), 226-245.

Kyle, A. (1985), 'Continuous auctions and insider trading', Econometrica 53(6), 1315-1335.

LeBaron, B., Arthur, W. and Palmer, R. (1999), 'Time series properties of an artificial stock market.', Journal of Economic Dynamics and Control 23, 1487-1516.

LeBaron, B. and Yamamoto, R. (2008), 'The impact of imitation on long-memory in an orderdriven market', Eastern Economic Journal 34, 504-517.

Lettau, M. and Uhlig, H. (1999), 'Rules of thumb versus dynamic programming', American Economic Review 89, 148-174.

Marimon, R., McGrattan, E. and Sargent, T. J. (1990), 'Money as a medium of exchange in an economy with artificially intelligent agents', Journal of Economic Dynamics and Control 14, 329-373.

Menkhoff, L., Osler, C. L. and Schmeling, M. (2010), 'Limit-order submission strategies under asymmetric information', Journal of Banking 8 Finance 34, 2665-2677.

Nicolosi, G., Peng, L. and Zhu, N. (2009), 'Do individual investors learn from their trading experience?', Journal of Financial Markets 12, 317-336.

Nyholm, K. (2003), 'Inferring the private information content of trades: A regime-switching approach', Journal of Applied Econometrics 18, 457-470.

O'Hara, M. (2001), 'Overview: Market structure issues in market liquidity', in Market liquidity: proceedings of a workshop held at the BIS', Bank for International Settlements (BIS) Papers. 
Parlour, C. A. and Seppi, D. J. (2008), 'Limit order markets: a survey', in Handbook of Financial Intermediation and Banking, Eds. Boot, A.W.A. and Thakor, A.V., North-Holland, pp. 63-96.

Pouget, S. (2007), 'Adaptive traders and the design of financial markets', Journal of Finance 62, 2835-2864.

Putniṇš, T. J. and Michayluk, D. (2013), Liquidity provision in limit order book markets, Working paper, SSRN.

Rosu, I. (2012), ' Order Choice and Information in Limit Order Markets', in Market Microstructure: Confronting Many Viewpoints, Eds. Abergel, F. and Bouchaud,J. and Foucault, T. and Lehalle, C.A. and Rosenbaum, M., Wiley, pp. 41-60.

Rosu, I. (2014), 'Liquidity and information in order driven markets', Working paper, SSRN.

Routledge, B. (2001), 'Genetic algorithm learning to choose and use information', Macroeconomic Dynamics 5, 303-325.

Routledge, B. R. (1999), 'Adaptive learning in financial markets', Review of Financial Studies 12, 1165-1202.

Ruchti, T. G. (2012), Estimating an equilibrium model of limit order markets, Working paper, Division of Humanities and Social Sciences, Caltech.

Seppi, D. J. (1994), 'Liquidity provision with limit orders and a strategic specialist', Review of Financial Studies 10, 103-150.

Theissen, E. (2000), 'Market structure, informational efficiency and liquidity: An experimental comparison of auction and dealer markets', Journal of Financial Markets 3(4), 333-363.

Wei, L., Zhang, W., He, X. and Zhang, Y. (2013), 'The value of learning and liquidity in limit order markets, Working paper 333, Quantitative Finance Research Centre (QFRC), University of Technolgy Sydney.

Yan, Y. and Zhang, S. (2012), 'An improved estimation method and empirical properties of the probability of informed trading', Journal of Banking \&3 Finance 36(2), 454-467. 